The Astrophysics Journal, 779, 110 (17PP), 2013, DeCEMBer 20

Preprint typeset using L LTEX style emulateapj v. 08/22/09

\title{
A BAYESIAN APPROACH TO ESTIMATE THE SIZE AND STRUCTURE OF THE BROAD-LINE REGION IN ACTIVE GALACTIC NUCLEI USING REVERBERATION MAPPING DATA
}

\author{
YAN-RONG Li ${ }^{1}$, JiAn-Min WANG ${ }^{1,2}$, LUIS C. Ho ${ }^{3,4}$, PU DU ${ }^{1}$, AND Jin-Ming BAI ${ }^{5,6}$ \\ published 2013 November 27
}

\begin{abstract}
This is the first paper in a series devoted to systematic study of the size and structure of the broad-line region (BLR) in active galactic nuclei (AGNs) using reverberation mapping (RM) data. We employ a recently developed Bayesian approach that statistically describes the variability as a damped random walk process and delineates the BLR structure using a flexible disk geometry that can account for a variety of shapes, including disks, rings, shells, and spheres. We allow for the possibility that the line emission may respond non-linearly to the continuum, and we detrend the light curves when there is clear evidence for secular variation. We use a Markov Chain Monte Carlo implementation based on Bayesian statistics to recover the parameters and uncertainties for the BLR model. The corresponding transfer function is obtained self-consistently. We tentatively constrain the virial factor used to estimate black hole masses; more accurate determinations will have to await velocity-resolved RM data. Application of our method to RM data with $\mathrm{H} \beta$ monitoring for about 40 objects shows that the assumed BLR geometry can reproduce quite well the observed emission-line fluxes from the continuum light curves. We find that the $\mathrm{H} \beta$ BLR sizes obtained from our method are on average $~ 20 \%$ larger than those derived from the traditional cross-correlation method. Nevertheless, we still find a tight BLR size-luminosity relation with a slope of $\alpha=0.55 \pm 0.03$ and an intrinsic scatter of $\sim 0.18 \mathrm{dex}$. In particular, we demonstrate that our approach yields appropriate BLR sizes for some objects (such as Mrk 142 and PG 2130+099) where traditional methods previously encountered difficulties.
\end{abstract}

Subject headings: galaxies: active — methods: data analysis — methods: statistical — quasars: general

\section{INTRODUCTION}

The well-established technique of reverberation mapping (RM) provides a promising pathway for directly measuring black hole mass in active galactic nuclei (AGNs) with broad emission lines (Blandford \& McKee 1982; Peterson 1993, 2013). Efforts over the past two decades have yielded RM measurements for $\sim 50$ nearby Seyfert galaxies and quasars (e.g., Bentz et al. 2013) and led to the discovery of the widely used relationship between the size of the broad-line region (BLR) and the optical luminosity of the AGN (Kaspi et al. 2000, 2005; Vestergaard \& Peterson 2006; Bentz et al. 2009a, 2013), which serves as a cornerstone to study the demography of supermassive black holes in large AGN surveys (e.g., Greene \& Ho 2007; Shen et al. 2008; Vestergaard \& Osmer 2009) and secondary explorations of the role of supermassive black holes in various astrophysical contexts (e.g., Marconi et al. 2004; Ho 2008; Wang et al. 2009; Li et al. 2011, 2012).

The underlying principle of the RM technique is quite straightforward. Emission line variations are blurred echoes of continuum variations through the transfer function, which encodes the geometry and kinematic information of the BLR. On both theoretical and observational side, much attention

1 Key Laboratory for Particle Astrophysics, Institute of High Energy Physics, Chinese Academy of Sciences, 19B Yuquan Road, Beijing 100049, China; liyanrong@mail.ihep.ac.cn

2 National Astronomical Observatories of China, Chinese Academy of Sciences, 20A Datun Road, Beijing 100020, China

3 Kavli Institute for Astronomy and Astrophysics, Peking University, Beijing 100871, China

4 The Observatories of the Carnegie Institution for Science, 813 Santa Barbara Street, Pasadena, CA 91101, USA

5 National Astronomical Observatories/Yunnan Observatory, Chinese Academy of Sciences, Kunming 650011, China

6 Key Laboratory for the Structure and Evolution of Celestial Objects, Chinese Academy of Sciences, Kunming 650011, China has been paid to recovering the transfer function or velocitydelay map with the purpose of placing constraints on the basic properties and structure of the BLR (e.g., Horne et al. 1991; Welsh \& Horne 1991; Wanders et al. 1995; Ulrich \& Horne 1996; Kollatschny 2003; Denney et al. 2009; Bentz et al. 2010; Goad et al. 2012; Grier et al. 2013b). Traditional cross-correlation analysis, used in most previous reverberation studies, has succeeded in characterizing BLR sizes using the derived time lags (e.g., Bentz et al. 2013 and references therein), although their interpretation in terms of realistic BLR structures remains elusive (Netzer 1990; Robinson \& Perez 1990; Welsh 1999). There are other more sophisticated mathematical methods developed for reconstructing the transfer function, including the maximum entropy technique (Horne 1994), the regularized linear inverse method (Krolik \& Done 1995), and the SOLA method (Pijpers \& Wanders 1994). A physical model for the BLR is finally invoked to decode the transfer functions (e.g., Horne et al. 2003; Bentz et al. 2010; Grier et al. 2013b).

A major concern of these traditional methods is the assumption that the emission lines respond linearly to the ionizing continuum. This is the case for optically thin BLRs. However, for optically thick BLRs, while the total line and diffuse continua emission are proportional to the continuum flux, the emission of individual lines might not. Photoionization calculations show that the responses of different emission lines depend on the ionization parameter (e.g., Netzer et al. 1985). The situation is even more complicated when using the $5100 \AA$ A continuum luminosity instead of the unobservable UV ionizing continuum for RM analysis. The shape of the incident continuum most likely does not remain constant during the reverberation variations. Such evidence has been found in long-term RM monitoring of the well-studied Seyfert galaxy NGC 5548 (Dietrich \& Kollatschny 1995; Peterson et 
al. 2002; Bentz et al. 2007). Moreover, it is common for the variation amplitude of the emission lines to exceed that of the optical continuum (e.g., Meusinger et al. 2011 and references therein). This is difficult to reconcile in the framework of linear response.

On the other hand, due to our ignorance of the structure and kinematics of the BLR, a virial factor $\left(f_{\mathrm{BLR}}\right)$ has to be assumed to convert the observed emission line widths and reverberation time lags into black hole mass. In the absence of any other direct black hole mass measurements, a common practice is to calibrate $f_{\mathrm{BLR}}$ with the aid of the relationship between the black hole mass and stellar velocity dispersion of the bulge of the host galaxy $\left(M_{\bullet}-\sigma_{\star}\right.$ relation; Onken et al. 2004; Woo et al. 2010; Park et al. 2013), which is well established in local quiescent galaxies. The average value of $f_{\mathrm{BLR}}$ derived in the literature ranges from $\left\langle f_{\mathrm{BLR}}\right\rangle \approx 3$ (Marconi et al. 2008; Graham et al. 2011) up to $\sim 6$ (Onken et al. 2004; Woo et al. 2010; Grier et al. 2013a) with a scatter of about 0.4 dex, comparable to (Gültekin et al. 2009) or slightly larger than (Kormendy \& Ho 2013) that of the $M_{\bullet}-\sigma_{\star}$ relation. The virial factor calibrated in this manner is valid only in a statistical sense. It seems likely that $f_{\mathrm{BLR}}$ differs from object to object (see the extensive discussion of Goad et al. 2012). The dynamical timescale of the BLR, $R_{\mathrm{BLR}} / \Delta V$, is on the order of a few years for typical values of $R_{\mathrm{BLR}} \approx 10$ light days and $\Delta V \approx 2000 \mathrm{~km} \mathrm{~s}^{-1}$. Long-term monitoring of NGC 5548 demonstrates that its BLR structure evolves year-to-year (Wanders \& Peterson 1996; Peterson et al. 2002; Shapovalova et al. 2004; Sergeev et al. 2007), potentially implying that $f_{\mathrm{BLR}}$ is also subject to variations. Furthermore, Pancoast et al. (2011) recently developed a fully general Bayesian framework to analyze RM data sets to model the geometry and dynamics of the BLR. Subsequent application of this technique on Mrk 50 yields a virial factor $f_{\mathrm{BLR}} \approx 6$ (Pancoast et al. 2012), consistent with the nominal value commonly adopted, but for Arp 151 the value of $f_{\mathrm{BLR}} \approx 2.5$ seems somehow exceptional (Brewer et al. 2011). In this regard, an object-byobject determination of $f_{\mathrm{BLR}}$ will help to better understand the mass measurements and will permit a comprehensive exploration of BLR structures.

Following the Bayesian framework developed by Pancoast et al. (2011), this paper is devoted to systematically study the structure of the BLR using RM data sets with $\mathrm{H} \beta$ monitoring accessible in the literature. Compared to the traditional methodology through the transfer function or velocity-delay maps, the Bayesian approach enables a direct probe of the BLR structure and a routine estimate of the modeling parameters. We take into account the non-linear response of emission lines to continuum variations, and we study the effect of detrending to remove secular variations that may contaminate the RM analysis. We attempt to recover the BLR geometry and place tentative constraints on the virial factors. We describe the methodology of our approach in Section 2 and the sample of RM light curves compiled from the literature in Section 3. In Section 4, we present a general estimate to the virial factor for our assumed BLR geometry. Section 5 presents verifications of our approach, and our results are given in Section 6. We discuss the uncertainties of our method and future improvements in Section 7. The conclusions are summarized in Section 8.

Throughout the paper, we adopt a standard $\Lambda$ CDM cosmology with $\Omega_{m}=0.3, \Omega_{\Lambda}=0.7$, and $H_{0}=72 \mathrm{~km} \mathrm{~s}^{-1} \mathrm{Mpc}^{-1}$. Unless stated otherwise, the BLR sizes derived from time series analysis always refer to rest-frame values (at redshift $z$ ); this and other variables invoking cosmic time are reduced by a factor of $(1+z)$ with respect to the values in the observer's frame. For the sake of brevity, when referring to the Julian Date, only the five least significant digits are retained.

\section{METHODOLOGY}

\subsection{Continuum Variability and Reconstruction}

The first step in analysis of RM data is reconstructing the continuum light curve from usually irregularly sampled data. For this purpose, we adopt and slightly modify the framework outlined by Rybicki \& Press (1992). For the sake of comparison, the notation used here also follows Rybicki \& Press (1992). Detailed derivations of the following equations are given in Appendix A.

Let the column vector $\boldsymbol{y}$ denote a set of $m$ measurements for a light curve in a monitoring campaign. In practice, each measurement $y_{i}$ can be deemed to be the sum of an underlying signal $s_{i}$ representing the variation, a constant $q$ representing the mean of the light curve, and a noise value $n_{i}$ representing the associated measurement error. Written in a concise form of column vectors,

$$
\boldsymbol{y}=\boldsymbol{s}+\boldsymbol{n}+\boldsymbol{E} q
$$

where $\boldsymbol{E}$ is a vector with all unity elements (i.e. $E_{i}=1$ ). We will demonstrate below that it is particularly necessary to separate out the mean before performing the reconstruction.

Without any independent statistical information about the signal $s$, a practical strategy is to assume that $s$ is stationary. This simplifies the covariance function of $s$, denoted by $S\left(t_{1}, t_{2}\right)$, between times $t_{1}$ and $t_{2}$ in a way that $S\left(t_{1}, t_{2}\right)$ depends only on the time difference $t_{1}-t_{2}$ (Rybicki \& Press 1992). Recent work by Kelly et al. (2009) finds that the covariance function driven by a damped random walk model, expressed as

$$
S\left(t_{1}, t_{2}\right)=\sigma_{\mathrm{d}}^{2} \exp \left[-\left(\frac{\left|t_{1}-t_{2}\right|}{\tau_{\mathrm{d}}}\right)^{\alpha}\right]
$$

can well describe the optical variability of AGNs. This is further reinforced by subsequent investigations of large samples of AGN light curves (Kozłowski et al. 2010; MacLeod et al. 2010, 2011; Kelly et al. 2011; Zu et al. 2013). Here, $\tau_{\mathrm{d}}$ is the typical timescale of variation, $\sigma_{\mathrm{d}}$ is the standard deviation of variation on long-timescale $\left(\gg \tau_{\mathrm{d}}\right)$, and $\alpha$ is a smoothness parameter. Previous studies show that $\alpha=1$ is sufficient for interpreting the variabilities (MacLeod et al. 2010; $\mathrm{Zu}$ et al. 2013), and we therefore fix it throughout the calculations.

If we further assume that both $s$ and $\boldsymbol{n}$ are Gaussian and uncorrelated, the probability for a realization of $y$ is (see Appendix A for details)

$$
P\left(\boldsymbol{y} \mid \sigma_{\mathrm{d}}, \tau_{\mathrm{d}}\right)=\frac{1}{\sqrt{(2 \pi)^{m}|\boldsymbol{C}|}} \exp \left\{-\frac{1}{2}(\boldsymbol{y}-\boldsymbol{E} \hat{q})^{T} \boldsymbol{C}^{-1}(\boldsymbol{y}-\boldsymbol{E} \hat{q})\right\},
$$

where superscript " $T$ " denotes the transposition, $C \equiv S+\boldsymbol{N}$, $S$ is the covariance matrix of signal $s$ given by Equation (2), $\boldsymbol{N}$ is the covariance matrix of the noise $\boldsymbol{n}$, and the best estimate of $q$ is (see also Rybicki \& Press 1992)

$$
\hat{q}=\frac{\boldsymbol{E}^{T} \boldsymbol{C}^{-1} \boldsymbol{y}}{\boldsymbol{E}^{T} \boldsymbol{C}^{-1} \boldsymbol{E}}
$$

Now, given a set of measurement, we recover the damped 


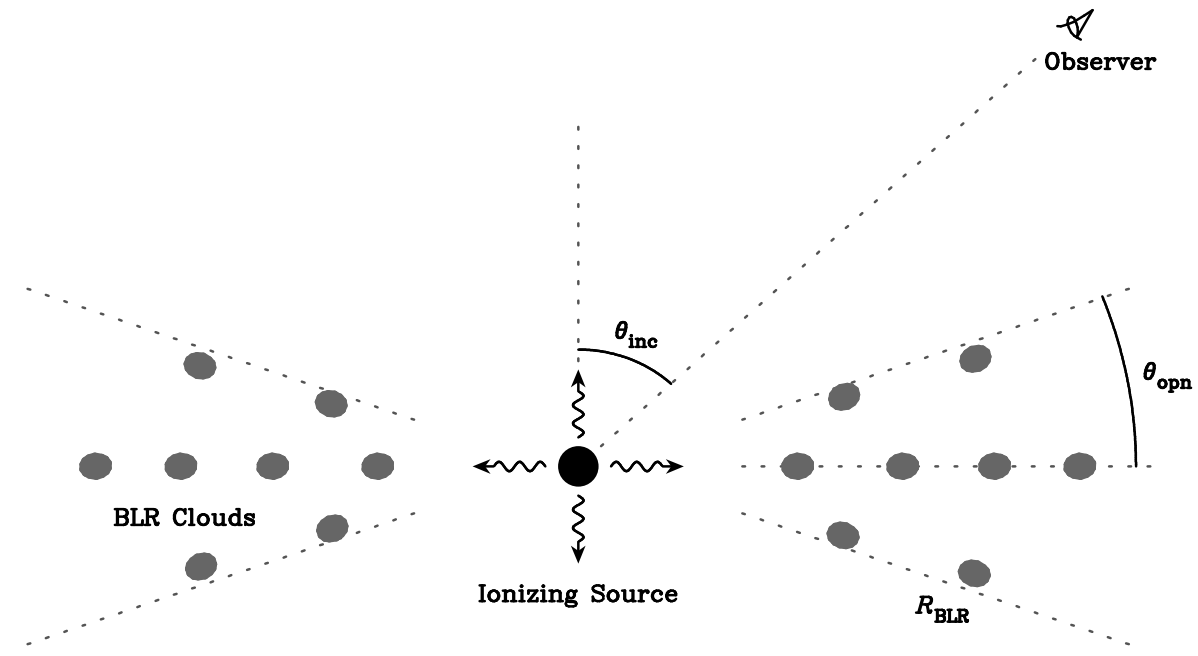

FIG. 1.- Schematic of the BLR geometry. The BLR has a flexible disk-like geometry with an inclination angle $\theta_{\text {inc }}$ to the observer and an opening angle $\theta_{\text {opn }}$. A central ionizing source is assumed to produce an isotropic UV emission that illuminates the surrounding BLR clouds (see Section 2.2 for details).

random walk process with the aid of Bayes' theorem:

$$
\begin{aligned}
& P\left(\sigma_{\mathrm{d}}, \tau_{d} \mid \boldsymbol{y}\right)=\frac{P\left(\sigma_{\mathrm{d}}, \tau_{\mathrm{d}}\right) P\left(\boldsymbol{y} \mid \sigma_{\mathrm{d}}, \tau_{\mathrm{d}}\right)}{P(\boldsymbol{y})} \\
= & \frac{P\left(\sigma_{\mathrm{d}}, \tau_{\mathrm{d}}\right)}{\sqrt{(2 \pi)^{m}|\boldsymbol{C}|}} \exp \left\{-\frac{1}{2}(\boldsymbol{y}-\boldsymbol{E} \hat{q})^{T} \boldsymbol{C}^{-1}(\boldsymbol{y}-\boldsymbol{E} \hat{q})\right\},
\end{aligned}
$$

where the marginal likelihood $P(\boldsymbol{y})$ is merely a normalization factor that is neglected (Sivia \& Skilling 2006). Maximizing this posterior distribution yields best estimates for $\sigma_{\mathrm{d}}$ and $\tau_{\mathrm{d}}$. Specifically, we assign logarithmic priors for $\sigma_{\mathrm{d}}$ and $\tau_{\mathrm{d}}$ in Equation (5), first employ the simulated annealing algorithm (Liu 2001) to locate the solutions maximizing Equation (5), and then enter a Markov chain Monte Carlo analysis to explore the statistical properties of $\sigma_{\mathrm{d}}$ and $\tau_{\mathrm{d}}$.

After determining the best values for $\sigma_{\mathrm{d}}$ and $\tau_{\mathrm{d}}$, the most probable estimate of the light curve at any time $t_{\star}$ is (see Appendix A)

$$
\hat{y}_{\star}=S_{\star}^{T} C^{-1}(\boldsymbol{y}-\boldsymbol{E} \hat{q})+\hat{q},
$$

where $S_{\star}$ is a vector of the covariances between $t_{\star}$ and the time of each measurement point (given by Equation (2)). The mean square residual of this estimate is

$$
\left\langle\Delta \hat{y}_{\star}^{2}\right\rangle=\sigma_{\mathrm{d}}^{2}-\boldsymbol{S}_{\star}^{T} \boldsymbol{C}^{-1} \boldsymbol{S}_{\star}+\frac{\left(\boldsymbol{S}_{\star}^{T} \boldsymbol{C}^{-1} \boldsymbol{E}-1\right)^{2}}{\boldsymbol{E}^{T} \boldsymbol{C}^{-1} \boldsymbol{E}} .
$$

From Equation (2), we note that at gaps far from any data points the covariance matrix $S_{\star}$ approaches zero; thus, the estimate given by Equation (6) tends toward the mean of the light curve with its uncertainty increasing up to the standard deviation of the variation $\left(\sigma_{\mathrm{d}}\right)$. Meanwhile, if the mean $q$ of the light curve is not separated out as in Equation (6), the best estimate $\hat{y}$ tends toward zero at data gaps and hence give rise to a bias (see discussion in Rybicki \& Press 1992).

As shown by Rybicki \& Press (1992), a typical reconstruction of the observed light curve is obtained by adding to the most probable estimate $\hat{y}_{\star}$ a Gaussian random process with zero mean and covariance matrix $\left(\boldsymbol{S}^{-1}+\boldsymbol{N}^{-1}\right)^{-1}$.

It is worth mentioning that in the damped random walk model the variation of the light curve on short timescales $\left(t \ll \tau_{\mathrm{d}}\right)$ is $\sim \sigma_{\mathrm{d}} \sqrt{2 t / \tau_{\mathrm{d}}}$ (Kelly et al. 2009). Therefore, one can roughly obtain an order-of-magnitude estimate of $\tau_{\mathrm{d}}$ by simple inspection of the amount of variation. Larger $\tau_{\mathrm{d}}$ leads to slower variations and smoother light curves. Also, the parameters $\tau_{\mathrm{d}}$ and $\sigma$ are found to be correlated with the physical properties of accretion disks, including optical luminosities, Eddington ratios, and black hole masses (Kelly et al. 2009; MacLeod et al. 2011). Dexter \& Agol (2011) demonstrate the ability of the damped random walk model for accretion disk fluctuations to explain the microlensing observations of accretion disks.

\subsection{Broad-line Region Modeling}

A number of lines of evidence suggest that the BLR has a flattened shape: (1) emission lines with disk-like (e.g., double-peaked) profiles are common in AGNs (e.g., Eracleous \& Halpern 2003; Strateva et al. 2003; Gezari et al. 2007; Lewis et al. 2010 and references therein); (2) Balmer line widths correlate with the orientation of the rotation axis of the BLR (e.g., Rokaki et al. 2003; Jarvis \& McLure 2006; Decarli et al. 2008); (3) possible detection of orbital motion of the BLR (e.g., Sergeev et al. 2000; Eracleous et al. 2009). Furthermore, a number of previous velocity-resolved RM studies show the BLR to be disk-like (e.g., Bentz et al. 2010; Grier et al. 2013b; Peterson 2013). Motivated by these results, it is reasonable at this stage to assume that the BLR has a disklike structure. We follow Pancoast et al. (2011)'s approach, which has been successfully applied to the RM data of Arp 151 (Brewer et al. 2011) and Mrk 50 (Pancoast et al. 2012). The details of the modeling can be found in these original works. We here describe the essential points and our improvements thereof.

Figure 1 shows a schematic of the BLR geometry adopted in the present work. The BLR is represented by a large number of discrete, point-like clouds, which orbit around the central black hole and absorb the ionizing continuum from the central source (i.e. accretion disk) and re-radiate emission lines. The distribution of the clouds is a flexible axisymmetric disk, which, with suitable parameters, can account for a variety of shapes, including shells, spheres, and rings. The radial distribution of clouds is parameterized according to

$$
R=F \mu+(1-F) \mathscr{R},
$$

where $\mathscr{R}$ is a random variable drawn from a Gamma distribution with a mean $\mu$ and a standard deviation $\beta \mu$. In this con- 
figuration, the overall mean radius for the cloud distribution is $\mu$, the inner hard edge of the BLR is $R_{\text {in }}=F \mu$, and the mean radial width of the BLR is $\sigma_{R} \approx \mu \beta(1-F)$. The parameter $\beta$ controls the shape of the cloud distribution: small values of $\beta$ create narrow normal distributions, while large values tend to create exponential distributions. The value of $F$ lies in a range $0-1$. According to the previous works of Brewer et al. (2011) and Pancoast et al. (2012), it is adequate to set the range of $\beta$ to $0-1$.

The BLR clouds subtend a solid angle denoted by an opening angle $\theta_{\text {opn }}$, which is defined so that $\theta_{\text {opn }}$ approaching zero creates thin disks/rings and $\theta_{\text {opn }}$ approaching $\pi / 2$ creates spheres/shells. Within the opening angle, we assume that the clouds are uniformly distributed over the polar and azimuthal directions. Lastly, the BLR is viewed at an inclination angle $\theta_{\text {inc }}$ to the distant observer, which is defined so that an inclination of zero corresponds to face-on, and an inclination of $\pi / 2$ corresponds to edge-on. We give some specific cases for illustration purposes: For $\theta_{\text {opn }}=90^{\circ}$, one obtains a thin spherical shell with $F \rightarrow 1$ and $\beta \rightarrow 0$ and a thick sphere with $F \rightarrow 0$ and $\beta \rightarrow 1$.

Before producing the observed emission-line fluxes, we list a necessary ansatz: (1) The UV ionizing continuum is simply proportional to the optical $5100 \AA$ continuum; however, we relax the usual assumption of linear response of the emission lines (see also the early work by Gaskell \& Sparke 1986). (2) The ionizing source has a point-like geometry so that its emission is isotropic and falls off with the square of the distance. (3) All BLR clouds have the same size and density, and there is no shadowing among clouds. Given a cloud distribution, we predict the emission-line flux response to the continuum at time $t$ by summing over the emission from all the clouds:

$$
f_{l}(t)=\sum_{i} \epsilon_{i}(t)=A \sum_{i} w_{i}\left[I_{i} \frac{f_{c}\left(t-\tau_{i}\right)}{R_{i}^{2}}\right]^{1+\gamma},
$$

where $\tau_{i}$ is the time lag of the re-radiation from the $i$ th cloud at distance $R_{i}$ to the central source, $A$ is a response coefficient, $w_{i}$ is the weight of the cloud in response to the continuum, $I_{i}$ is a flexible parameter describing any possible anisotropic effects and deviations from the inverse square decline of the continuum flux, and $\gamma$ denotes the non-linearity of the response. The uncertainties inherent from measurement errors of the continuum are

$$
\Delta f_{l}(t) \approx(1+\gamma) A \sum_{i} w_{i}\left(\frac{I_{i}}{R_{i}^{2}}\right)^{1+\gamma} f_{c}^{\gamma}\left(t-\tau_{i}\right) \Delta f_{c}\left(t-\tau_{i}\right),
$$

where the variation amplitude is assumed to be small (as in most cases) and a linear expansion is used.

Rewriting Equations (9) and (10) into a general form,

$$
f_{l}(t)=A \int \Psi(\tau) f_{c}^{1+\gamma}(t-\tau) \mathrm{d} \tau
$$

and

$$
\Delta f_{l}(t)=(1+\gamma) A \int \Psi(\tau) f_{c}^{\gamma}(t-\tau) \Delta f_{c}(t-\tau) \mathrm{d} \tau
$$

with the transfer function

$$
\Psi(\tau)=\sum_{i} \delta\left(\tau-\tau_{i}\right) w_{i}\left(\frac{I_{i}}{R_{i}^{2}}\right)^{1+\gamma}
$$

where $\delta(x)$ is the Dirac delta function. If we let $\gamma=0$ in Equation (11), one arrives at the traditional linear response.

At this stage, we focus on the variation of the light curves, and the absolute units of the light curves are no longer important; thus, the coefficient $A$ is a nuisance parameter. For simplicity, we also fix the weights to $w_{i}=1$ and neglect $I_{i}$ throughout the calculations. We only make use of the velocityunresolved RM data sets such that the velocity information of the clouds disappears in Equation (11); however, this can be readily added (see Brewer et al. 2011; Pancoast et al. 2011, 2012).

The role of the free parameters in shaping the light curves of the emission lines are as follows. The mean radius $\mu$ determines the overall time lags of the emission lines. Larger inclination $\theta_{\text {inc }}$ tends to broaden the transfer functions toward double peaks, while larger opening angle $\theta_{\text {opn }}$ tends to broaden the transfer functions toward top-hat. The parameters $\beta$ and $F$ jointly control the width of the BLR and therefore the width of the transfer function. In addition, $F$ sets a hard inner limit on the BLR. The parameter $\gamma$ determines the amplitude of response to the continuum variations. It is apparent that the parameters $\theta_{\text {inc }}$ and $\theta_{\text {opn }}$ are degenerate at some level, as are the parameters $\beta$ and $F$. In general, there is also degeneracy among other parameters, depending on the quality of the RM data. High-fidelity data sets, namely those with a fine sampling rate, higher signal-to-noise ratio, and velocity-resolved information, are beneficial to eliminate these degeneracies.

The terminology "BLR size" needs to be clarified a bit here. We introduce two definitions: mass-weighted average radius

$$
R_{m}=\frac{\sum_{i} m_{i} R_{i}}{\sum m_{i}}
$$

where $m_{i}$ is the mass of $i$ th cloud, and emissivity-weighted average radius

$$
R_{e}=\frac{\sum_{i} \epsilon_{i} R_{i}}{\sum_{i} \epsilon_{i}}
$$

Because BLR clouds are treated as point masses with the same density and size, $R_{m}$ is exactly equal to the mean radius of the cloud distribution. From Equation (9), it is apparent that the emissivity of each cloud is variable with time in response to the continuum variation, and hence the emissivity-averaged radius is weakly time-dependent ${ }^{7}$. It is convenient to use the time-averaged value for $R_{e}$ over the duration of the RM campaign. It is easy to verify that generally $R_{e} \leqslant R_{m}$ since the ionization flux most likely declines with radius. However, in our present modeling via Equation (8), the clouds are mostly distributed around the mean radius $\mu$, leading to $R_{e} \approx R_{m}$ in most cases. We therefore hereafter only use $R_{m}$ to refer to the BLR size.

\subsection{Detrending of Light Curves}

As shown by Welsh (1999), long-term secular variability is incidentally detected over the duration of the campaign, which is found to be uncorrelated with reverberation variations (Sergeev et al. 2007) and thus will bias the desired correlation analysis between the continuum and emission light curves. The continuum and emission lines may display different secular trends. Plausible causes are, but not limited to, the

\footnotetext{
7 Note that BLRs may undergo secular evolution, leading to mass/emissivity-averaged radius varying significantly on a timescale of years (e.g., in NGC 5548; Wanders \& Peterson 1996; Peterson et al. 2002).
} 
non-linear response mentioned in the previous section, variations in the shape of the ionization continuum, as well as secular evolution of BLR structure, which is independent of the ionization sources.

To remove the bias due to secular variability, we adopt a first-order polynomial to fit the light curve with its mean subtracted and then detrend the original light curve by removing the polynomial. The goal of subtracting the mean before fitting is to keep the mean of the light curve unchanged. Detrending is necessary only when there is evident secular variability, such that the continuum and the emission light curves exhibit different secular trends. In such cases, the results of fitting the predicted emission line fluxes to observations will be substantially improved after detrending. In practice, we apply the detrending when the fitting to the emission lines is judged to be poor, which we define as $\chi^{2} /$ dof $>1.5$ (see below for the definition of $\chi^{2}$ ). The choice of this limit is a bit arbitrary, but it suffices to identify potential cases for detrending. The detrending is deemed acceptable if $\chi^{2} /$ dof diminishes.

\subsection{Markov Chain Monte Carlo Implementation}

The measured RM data in hand are the line flux time series with associated errors $\left(y_{l}, \sigma_{l}\right)$ and the continuum flux time series with associated errors $\left(y_{c}, \sigma_{c}\right)$; in most cases, both are irregularly sampled. We first reconstruct the continuum time series using the damped random walk model described in Section 2.1. Given the geometry model of the BLR with parameter set $\boldsymbol{\theta}$, a reconstruction of the line flux from the continuum flux series can be made as described in the preceding section; we denote the reconstructed flux by $y_{p}\left(y_{c} \mid \boldsymbol{\theta}\right)$ with errors $\sigma_{p}$. Suppose that the probability distribution for the measurement errors are Gaussian and uncorrelated. The likelihood function can then be written as

$$
P(D \mid \boldsymbol{\theta})=\prod_{i=1}^{m} \frac{1}{\sqrt{2 \pi\left(\sigma_{l}^{2}+\sigma_{p}^{2}\right)}} \exp \left(-\frac{1}{2} \frac{\left[y_{l}-y_{p}\left(y_{c} \mid \boldsymbol{\theta}\right)\right]^{2}}{\sigma_{l}^{2}+\sigma_{p}^{2}}\right),
$$

where $D$ represents the measured data. Again, from Bayes' theorem, the posterior probability distribution for $\boldsymbol{\theta}$ is given by

$$
P(\boldsymbol{\theta} \mid D)=\frac{P(\boldsymbol{\theta}) P(D \mid \boldsymbol{\theta})}{P(D)},
$$

where the marginal likelihood $P(D)$ is a normalization factor that is irrelevant to the subsequent analysis. The prior probabilities $P(\boldsymbol{\theta})$ in Equation (17) are assigned as follows: for parameters whose typical value ranges are known, a uniform prior is assigned; otherwise, if the parameter information is completely unknown, a logarithmic prior is assigned (Sivia \& Skilling 2006). Among the seven free parameters, the priors for the mean BLR size $\mu$ and the response coefficient $A$ are set to be logarithmic, and the rest (inclination $\theta_{\text {opn }}$, opening angle $\theta_{\text {opn }}$, non-linearity parameter $\gamma$, and radial distribution parameters $\beta$ and $F$ ) are set to uniform.

We employ the Markov Chain Monte Carlo method with parallel tempering and the Metropolis-Hastings algorithm (e.g., Liu 2001) to construct samples from the posterior probability distribution, and then explore the statistical properties of the model parameters. The parallel tempering algorithm guards the Markov chain against being stuck in a local maximum and expedites its convergence to globally optimized solutions. Depending on the individual object, the free parame- ters are probably correlated with each other. Using the covariance matrix of free parameters will improve the efficiency of Monte Carlo sampling. We initially input a diagonal covariance matrix and recompute it every 10,000 steps based on the newly generated section of the Markov chain. Empirically, after one or two iterations, the correlation matrix turns out to be stable and the Markov chain rapidly converges. The Markov chain is run 50,000 steps in total with $10^{6}$ BLR clouds. Unless stated otherwise, the best estimates for the parameters are taken to be the expectation value of their distribution and the uncertainties are taken to be the standard deviation. Presently we are unable to evaluate the systematic uncertainties inherent in the models.

We also introduce a $\chi^{2}$ for the fitting of the emission lines according to Equation (16):

$$
\chi^{2}=\sum_{i=1}^{m} \frac{\left[y_{l}-y_{p}\left(y_{c} \mid \boldsymbol{\theta}\right)\right]^{2}}{\sigma_{l}^{2}+\sigma_{p}^{2}} .
$$

There are seven free parameters for BLR modelings. Accordingly, the reduced $\chi^{2}$ is calculated from $m-7$ degrees of freedom (dof) for light curves with $m$ observations. As described in the preceding section, $\chi^{2} /$ dof is used to determine whether detrending is necessary or acceptable.

\section{DATA SAMPLE}

We extract light curves of all objects with $\mathrm{H} \beta$ monitoring accessible in the literature to date ${ }^{8}$, mostly from the homogeneous compilations of Peterson et al. (2004) and Bentz et al. (2009a, 2013). We make use of the data that were designated by Bentz et al. (2013) as reliable (see their Table 13). The properties of all objects are summarized in Table 1. Bentz et al. (2009a, 2013) used surface brightness decomposition of Hubble Space Telescope images to measure the starlight contribution of the host galaxies to the optical luminosity of the central nuclei for most of the RM objects. We use their hostcorrected luminosities in order to redetermine the $R_{\mathrm{BLR}}-L$ relationship. The Lick AGN Monitoring Project (LAMP) published photometric $B$-band and $V$-band continuum light curves (Bentz et al. 2009b; Walsh et al. 2009); we use their $V$-band photometric light curves. The magnitudes are converted into fluxes and then normalized to unity for computational convenience. The following objects require special treatment or comments.

1. For Fairall 9, the sampling rate at $1390 \AA$ was much higher than that in the optical band. Since the two bands vary simultaneously (Rodriguez-Pascual et al. 1997; Santos-Lleó et al. 1997), we use the UV data at 1390 $\AA$ as a surrogate for the more poorly sampled optical data.

2. NGC 3227 is known to be one of the most heavily reddened objects in the RM sample. Bentz et al. (2013) corrected its optical luminosity at $5100 \AA$ by an extinction of 0.26 dex based on the reddening curve of Crenshaw et al. (2001).

3. 3C 120 was quite poorly sampled during the 1989-1996 campaign. Although the monitoring duration lasted as

8 The International AGN Watch project provides machine-readable data tables for several objects, which can be directly downloaded from its Web site (http://www.astronomy.ohio-state.edu/ agnwatch). 


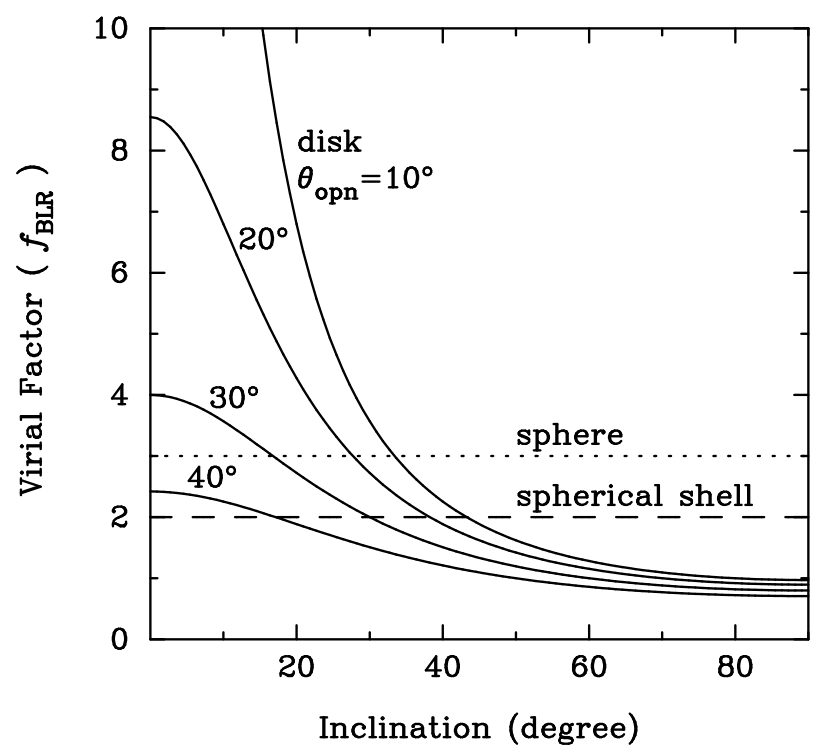

FIG. 2.- Dependence of the virial factor on inclination angle for different opening angles; see Equation (22). Dashed and dotted lines represent the virial factors for a spherical shell and sphere, respectively.

eight years, in total there were only 52 observations, with time gaps of about 100-300 days. To alleviate possible aliasing effects, we discard the beginning and ending parts of the light curves with particularly sparse sampling and only use the data between 1992 and 1996 (JD48869-JD50101).

4. The monitoring campaign of NGC 3783 was undertaken by the International AGN Watch (Stirpe et al. 1994). Onken \& Peterson (2002) recalibrated the optical spectra based on a refined algorithm. We use their revised data set for analysis.

5. NGC 7469 was monitored at MDM Observatory during 2010-2011 (Grier et al. 2012) but is missing from Table 1 because these data are not yet publicly accessible.

Peterson et al. (2004) reanalyzed the broad emission lines of all RM data available at that time and compiled line widths derived from the rms spectra. We directly use their measurements of $\mathrm{H} \beta$ line width for all the objects included in their compilation. For other objects, we quote line widths derived similarly from rms spectra given by the corresponding references listed in Table 1.

\section{THE VIRIAL FACTOR}

Assuming that the motion of BLR clouds is dominated by the gravity of the central black hole, the "virial" black hole mass can be obtained trivially through RM:

$$
M_{\mathrm{vir}}=f_{\mathrm{BLR}} \frac{V_{\mathrm{obs}}^{2} R_{\mathrm{BLR}}}{G},
$$

where $G$ is the gravitational constant and $V_{\text {obs }}$ is the emissionline velocity width. Here, the dimensionless so-called virial factor $f_{\mathrm{BLR}}$ subsumes all the unknown properties of the BLR, including its geometry, kinematics, and inclination angle. In the present approach, the BLR geometry is recovered statistically, thus allowing the virial factor to be estimated in an approximate sense (since the kinematics remains unknown at this stage).
For a thick Keplerian disk, the observed line width is related to the Keplerian velocity by (e.g., Collin et al. 2006)

$$
V_{\mathrm{obs}} \approx V_{\mathrm{Kep}}\left(h^{2}+\sin ^{2} \theta_{\mathrm{inc}}\right)^{1 / 2},
$$

where $h$ is the aspect ratio of the disk and $\theta_{\text {inc }}$ is the inclination angle. The aspect ratio is expressed in terms of the opening angle of the disk as $h \approx \sin \theta_{\text {opn }}$. Bear in mind that the line width measurement depends on the line profile and that there might be a scale factor of order of unity in Equation (20) (e.g., Collin et al. 2006). Combining Equation (19) with the true black hole mass estimate

$$
M_{\bullet}=\frac{V_{\mathrm{Kep}}^{2} R_{\mathrm{BLR}}}{G},
$$

the viral factor becomes

$$
f_{\mathrm{BLR}} \approx\left(\sin ^{2} \theta_{\mathrm{opn}}+\sin ^{2} \theta_{\mathrm{inc}}\right)^{-1} .
$$

Note that the above equation is valid only for a disk-like structure. When the opening angle approaches $90^{\circ}$, the BLRs become a spherical shell or a sphere. In such a case, if we further assume that the cloud motions are isotropic, the virial factor will be $f_{\mathrm{BLR}} \approx 2$ for thin shells and $f_{\mathrm{BLR}} \approx 3$ for spheres (Netzer 1990), independent of the inclination angle. Figure 2 illustrates the variation of the virial factor with inclination for different opening angles. As expected, the virial factor is highly sensitive to inclination for small opening angles and decreases with opening angle for a given inclination. In the following, we estimate $f_{\mathrm{BLR}}$ using Equation (22) if $\theta_{\mathrm{opn}}<40^{\circ}$; otherwise, we set $f_{\mathrm{BLR}} \approx 2-3$. Here $V_{\mathrm{obs}}$ refers to the velocity dispersion of the emission line, $\sigma_{\text {line }}$. If line width is parameterized instead by FWHM, the corresponding $f_{\mathrm{BLR}}$ drops roughly by a factor of four.

\section{VERIFICATIONS OF OUR APPROACH}

\subsection{An Illustration Case: Arp 151}

This object has been intensively studied in previous works based on the same RM data from the LAMP project (Bentz et al. 2009b; Walsh et al. 2009). Bentz et al. (2010) recovered the velocity-delay maps for multiple emission lines using the maximum-entropy technique and concluded that, although the constraints are not definitive, a plausible warped disk-like BLR is preferred. Brewer et al. (2011) used a Bayesian framework for velocity-resolved RM to directly measure the central black hole mass. Their results clearly suggest that a disk-like structure for the BLR can reproduce the observations quite well. They derived a virial factor $f_{\mathrm{BLR}}=2.5 \pm 1.6$ and a black hole mass $M_{\bullet}=(3.2 \pm 2.1) \times 10^{6} M_{\odot}$.

For the purpose of comparison, we plot our reconstruction of the continuum and $\mathrm{H} \beta$ line fluxes in the top panels of Figure 3. The typical rest-frame damping timescale for the damped random walk process is $\log \left(\tau_{\mathrm{d}} /\right.$ day $)=1.88 \pm 0.59$. The $\chi^{2} /$ dof of the fit to the $\mathrm{H} \beta$ line flux series is 0.88 . The top right panel of Figure 3 shows the recovered transfer function, which is remarkably consistent with the results from the maximum-entropy technique (see Figure 1 of Bentz et al. 2010). The best-fit values for the BLR parameters are $R_{\mathrm{d}}=4.0 \pm 0.4, \theta_{\text {inc }}=29^{\circ} \pm 18^{\circ}$, and $\theta_{\text {opn }}=36^{\circ} \pm 22^{\circ}$; these agree well with the model of Brewer et al. (2011) ${ }^{9}$. According

\footnotetext{
9 Note that Brewer et al. (2011) defined the inclination angle to be the complement of the usual convention adopted in the present calculations, and their opening angle is twice of that of our definition.
} 

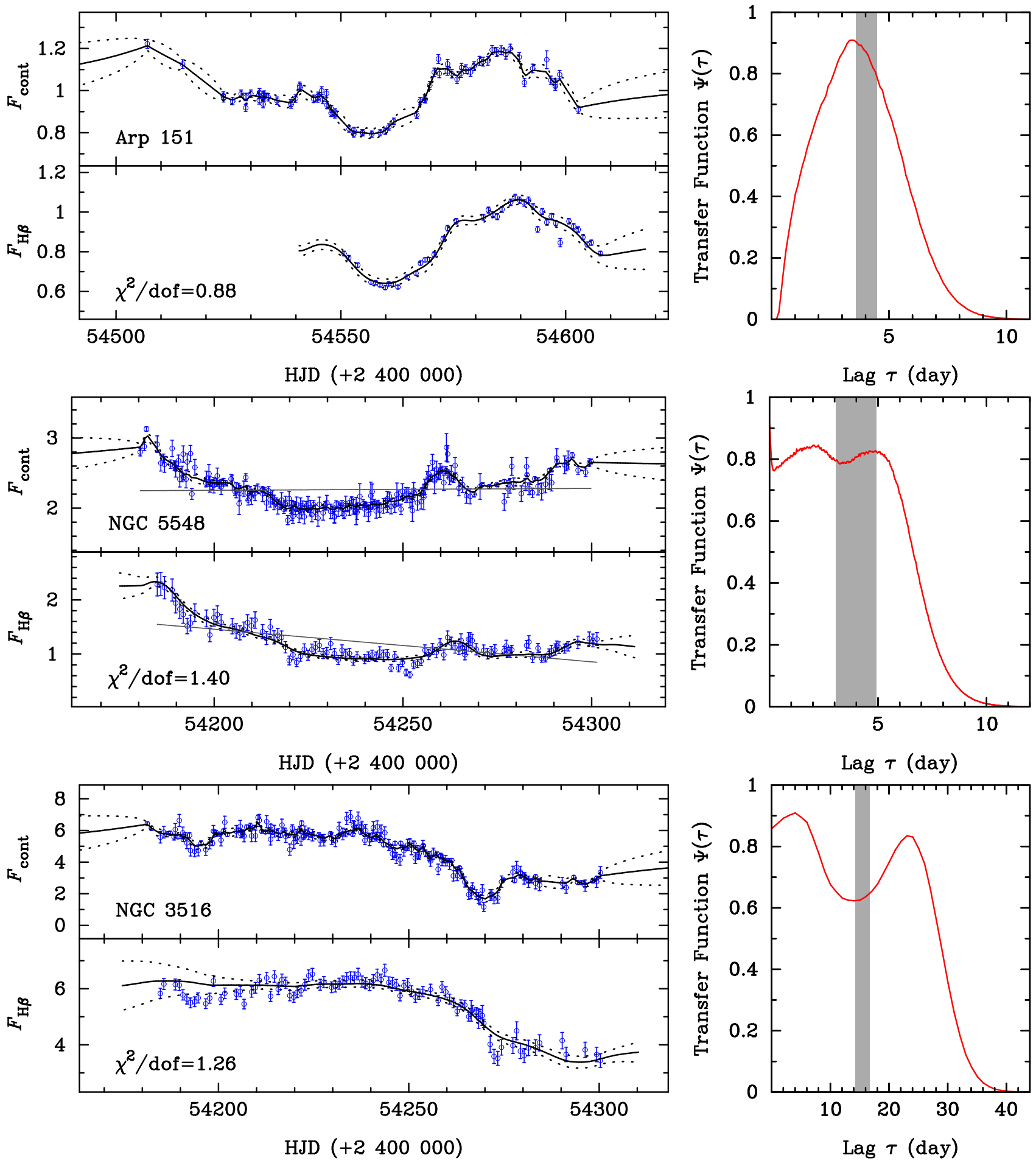

FIG. 3. - Three illustration cases: Arp 151, NGC 5548, and NGC 3516. (Left) Data points with error bars are the observed light curves. Thick solid lines show the reconstructed continuum at $5100 \AA$ (top panel) and the recovered $\mathrm{H} \beta$ emission (bottom panel); dashed lines represent the uncertainties. For NGC 5548 (middle panels), the gray line shows the fit for the detrending applied to remove secular variation. (Right) Transfer function $\Psi(\tau)$ (red curve, in arbitrary units) derived from the best-fit BLR model. The vertical shaded area represents the recovered BLR size and its uncertainty.

to Figure 2 , this gives a virial factor $f_{\mathrm{BLR}} \approx 1.8$, which implies that the simple estimate given by Equation (22) is quite acceptable and that, provided with high-quality RM data, even velocity-unresolved RM yields a viable measurement of black hole mass. We also emphasize that, as seen from the top right panel of Figure 2, the peak of the transfer function needs not coincide with the BLR size.
With a non-linearity parameter of $\gamma=0.3 \pm 0.04$, the BLR deviates slightly deviating linear response. Indeed, in support of this result, the excess variance of $\mathrm{H} \beta, F_{\text {var }}=0.169$, exceeds that of the $5100 \AA$ continuum flux, $F_{\text {var }}=0.120$ (see Table 8 of Bentz et al. 2009b). Here, the excess variance is defined as $F_{\text {var }}=\sqrt{\sigma_{F}^{2}-\delta_{F}^{2}} /\langle F\rangle$, where $\sigma_{F}^{2}$ is the variance of the observed 


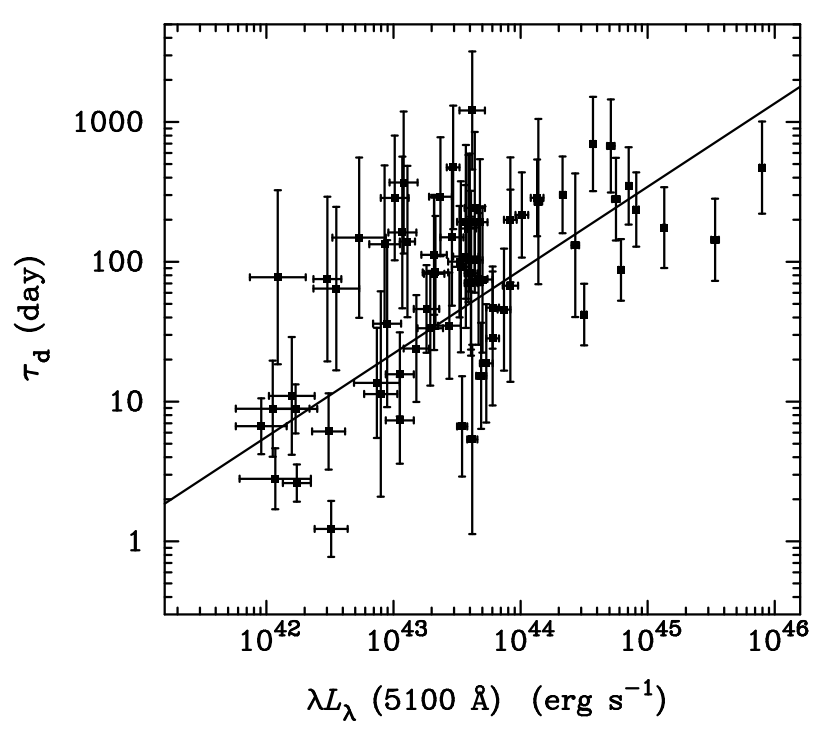

FIG. 4. - Variation of the damping timescale $\tau_{\text {d }}$ with optical (5100 ̊) AGN continuum luminosity. Solid line represents the best linear fit.

flux, $\delta_{F}^{2}$ is the mean square uncertainty, and $\langle F\rangle$ is the mean flux. $F_{\text {var }}$ represents the amplitude of flux variance of the light curve.

\subsection{Comparisons with Other Independent Measurements}

NGC 3227. NGC 3227 is a local Seyfert 1 galaxy, sufficiently close that its nuclear region is spatially resolved and dynamical modeling has been successfully applied to determine its central black hole mass. Using VLT SINFONI adaptive optics integral-field spectroscopy with a resolution of $\sim 7$ pc, Davies et al. (2006) derived a black hole mass in the range $(0.7-2) \times 10^{7} M_{\odot}$ from stellar dynamical modeling. Meanwhile, Hicks \& Malkan (2008) analyzed the kinematics of the molecular hydrogen gas in the central region of NGC 3227 by modeling the gas velocity field as a flat circular disk and reported a black hole mass of $2.0_{-0.4}^{+1.0} \times 10^{7} M_{\odot}$.

In our modeling of the RM data from the campaign at MDM observatory (Denney et al. 2010), the best value for the BLR size is $R_{\mathrm{d}}=5.1 \pm 0.7$ light days and the inclination angle is $\theta_{\text {inc }}=60^{\circ} \pm 22^{\circ}$. The opening angle is $\theta_{\text {opn }}=48^{\circ} \pm 24^{\circ}$, but it is not well recovered because the distribution is nearly uniform between $0^{\circ}$ and $90^{\circ}$. This geometry roughly leads to a virial factor $f_{\mathrm{BLR}} \approx 1-3$. As a result, the black hole mass for NGC 3227 lies in the range $(1.3-6.3) \times 10^{6} M_{\odot}$ using the $\mathrm{H} \beta$ line dispersion and $(2.2-10.6) \times 10^{6} M_{\odot}$ using FWHM. These results are marginally consistent with previous dynamical mass measurements. Moreover, the $\mathrm{H} \beta$ line in the rms spectra shows a prominent double-peak profile (see Figure 5 of Denney et al. 2010), strongly indicating a large inclination angle.

NGC 4151. NGC 4151 is another local Seyfert 1 galaxy with a measured central black hole mass. Hicks \& Malkan (2008) used gas kinematics to derive a mass of $3.0_{-2.2}^{+0.75} \times$ $10^{7} M_{\odot}$. The stellar dynamics analysis of Onken et al. (2007) gave an upper limit to the black hole mass of $<5 \times 10^{7} M_{\odot}$.

The latest monitoring campaign on NGC 4151 was carried out in 2006 using the 1.3-m telescope at MDM observatory (Bentz et al. 2006; see also Maoz et al. 1991; Kaspi et al. 1996). Our modeling gives $R_{\mathrm{d}}=7.9 \pm 0.9, \theta_{\mathrm{inc}}=53^{\circ} \pm 18^{\circ}$, and $\theta_{\mathrm{opn}}=57^{\circ} \pm 21^{\circ}$. For a virial factor of $f_{\mathrm{BLR}} \approx 1-3$, the black hole mass lies in a range of $(1.0-3.7) \times 10^{7} M_{\odot}$ using

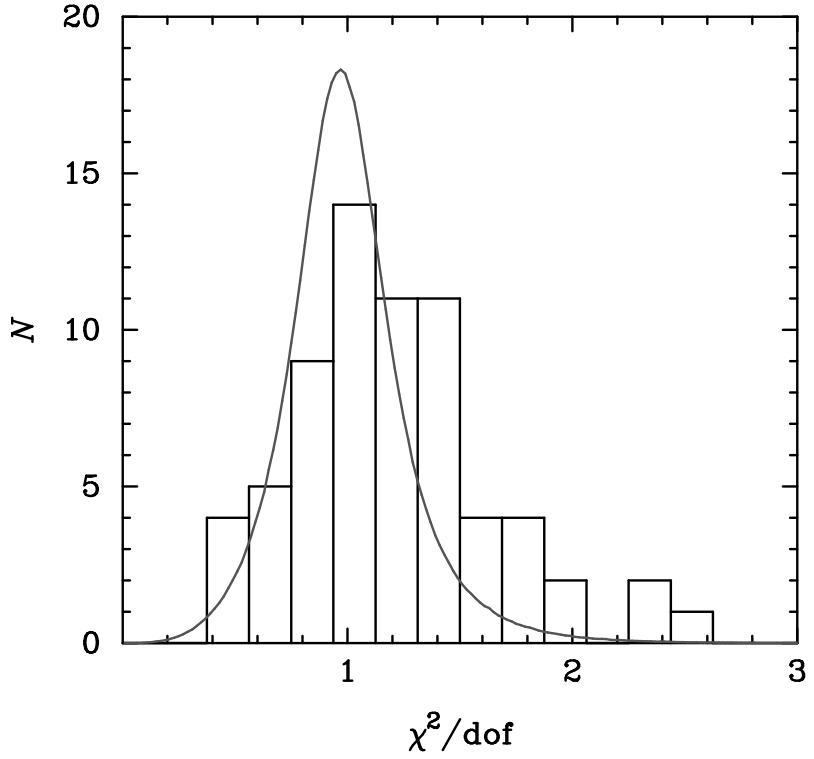

FIG. 5.- Distribution of reduced $\chi^{2}$ for the emission-line fits. Gray solid line represents the expected $\chi^{2}$ distribution.

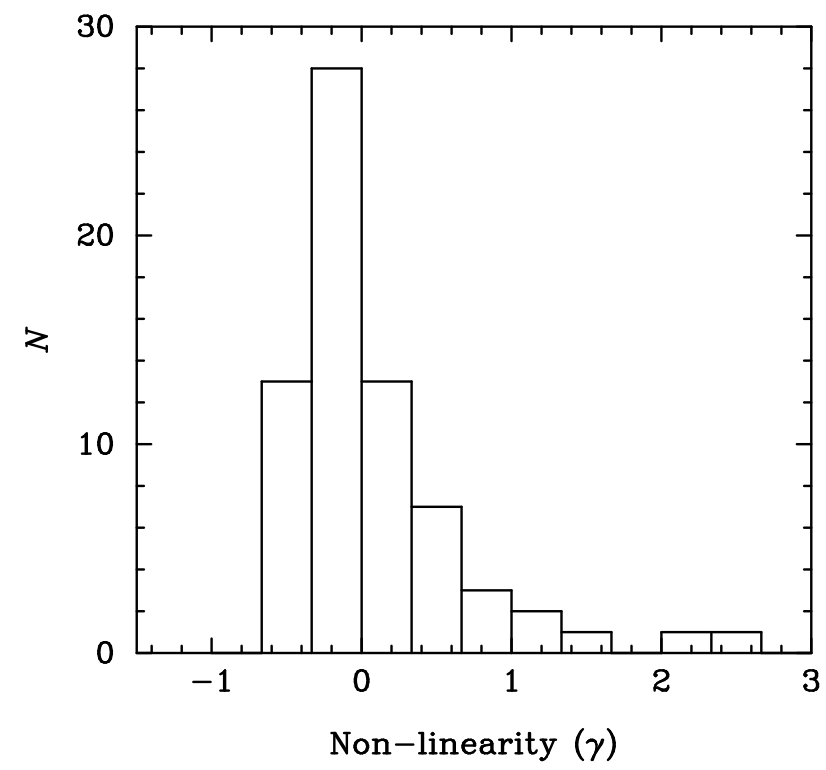

FIG. 6.- Distribution of the non-linearity parameter $\gamma$ for the emission-line response to the continuum.

$\sigma_{\text {line }}$ and $(0.7-2.8) \times 10^{7} M_{\odot}$ using FWHM. It is interesting to mention that the stellar velocity dispersion of NGC 4151 is $\sigma_{\star}=98 \mathrm{~km} \mathrm{~s}^{-1}$ (Onken et al. 2007), which yields a black hole mass of $1.4 \times 10^{7} M_{\odot}$ according to the $M_{\bullet}-\sigma_{\star}$ calibration of Kormendy \& Ho (2013).

In summary, the use of $\mathrm{H} \beta$ light curves alone does not yield an accurate determination of the virial factor. Nevertheless, tentative constraints on the virial factor yield black hole mass rough estimates compatible with independent mass estimates based on spatially resolved stellar and gas dynamical analysis. Future incorporation of line profiles will help to determine the virial factors more precisely.

\section{RESULTS}

\subsection{Overview}


In Figure 3, we also present illustration cases for NGC 5548 and NGC 3516. The fit to the $\mathrm{H} \beta$ light curve of NGC 5548 is remarkably good after detrending, as shown by the gray line. In particular, the small bump near JD54265 is well reproduced. The corresponding transfer functions for Arp 151, NGC 5548, and NGC 3516 have quite diverse shapes, ranging from a single peak, to roughly a top hat, to double peaks, respectively. For non-unimodal transfer functions like those of NGC 5548 and NGC 3516, the peak value of the transfer function cannot be used to represent the BLR size.

The recovered parameters for the BLRs of all the objects are summarized in Table 2. We tabulate the damping timescale $\tau_{\mathrm{d}}$ but omit the parameter $\sigma_{\mathrm{d}}$ for the damped random walk model because we pay no attention to the absolute units of the light curves. Of the seven free parameters for the BLR model, $\beta$ and $F$, which jointly determine the width of the BLR, are almost uniformly distributed over the specified ranges. This implies that BLR widths $\sigma_{R} \approx 0.3 R_{\mathrm{BLR}}$ can generally reproduce the observed $\mathrm{H} \beta$ light curves. The virial factors are calculated according to Equation (22) for $\theta_{\mathrm{opn}}<40^{\circ}$. The uncertainties are not given owing to the approximation underlying Equation (22). Overall, the virial factors range from $\sim 1$ up to $\sim 10$. Future velocity-resolved RM will better constrain the virial factors.

In Appendix B, we provide a supplementary online figure to summarize the reconstructions of the continua and emission lines, the derived transfer functions, and the distributions of BLR parameters for all the objects. We present the detailed results in following sections.

\subsection{Damped Random Walk Model for the Continuum}

Figure 4 shows the dependence of the rest-frame damping timescale for the damped random walk model for AGN variability on the luminosity at $5100 \AA$. As expected, the timescale $\tau_{\mathrm{d}}$ increases with luminosity (Kelly et al. 2009; MacLeod et al. 2010). A linear regression using the fitting algorithm FITEXY (Press et al. 1992, including a term for the intrinsic scatter; see Section 7 below for details), yields

$\log \left(\frac{\tau_{\mathrm{d}}}{\text { day }}\right)=(1.94 \pm 0.07)+(0.60 \pm 0.06) \log \left[\frac{\lambda L_{\lambda}(5100 \AA)}{10^{44} \mathrm{erg} \mathrm{s}^{-1}}\right]$.

A previous study by Kelly et al. (2009) found a similar relation using a sample composed of $\sim 55$ high-redshift AGNs from the MACHO survey and 45 nearby AGNs. Interestingly, Kelly et al. (2009) claimed that this relationship is consistent with that for the orbital or thermal timescale of the accretion disks, and thus a constraint on the viscosity parameter can be obtained. Given the feasibility of obtaining robust mass measurements from the present RM sample, a comprehensive investigation of the correlation between the parameters of damped random walk model with black hole mass will provide insights into the physics of accretion disks and the origins of their variability (Dexter \& Agol 2011).

\subsection{Response of Emission Lines}

Figure 5 presents the distribution of the reduced $\chi^{2}$ for the emission-line fits using the best recovered parameter set. The expected distribution of $\chi^{2} / \mathrm{dof}$ is also superposed for comparison. There is a moderate discrepancy for $\chi^{2} /$ dof larger that $\sim 1.5$; this is probably due to systematic errors (e.g., calibration errors) that may not included in the reported errors of the original data (e.g., NGC 4051 and PG 0052+251).

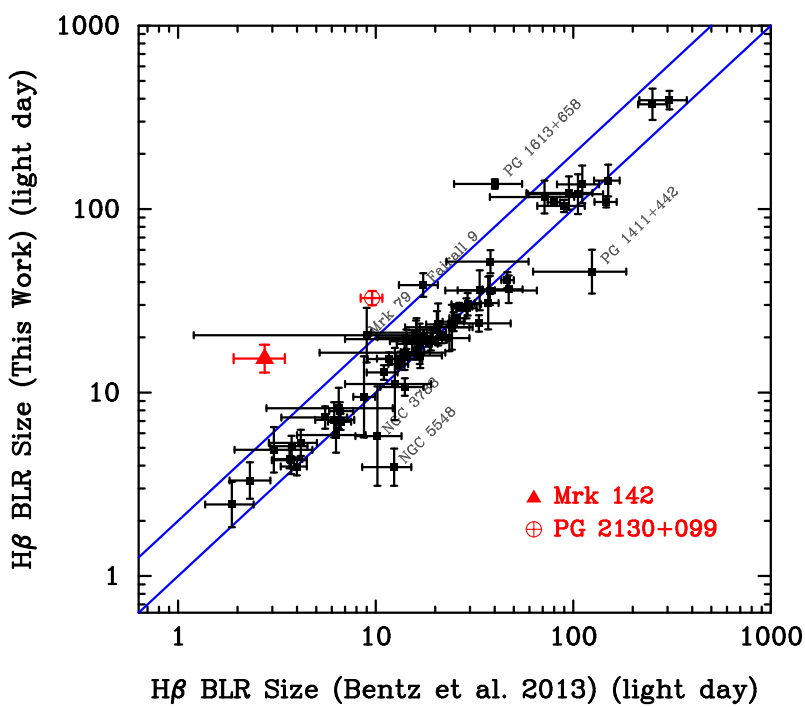

FIG. 7.- Comparison of the $\mathrm{H} \beta$ BLR size derived from this work with that from Bentz et al. (2013). The two solid lines represent $y=x$ and $y=2 x$, respectively.

Among the set of 70 light curves analyzed, the fits are especially poor $\left(\chi^{2} /\right.$ dof $\left.>3\right)$ for PG $1613+658$ and Mrk 79 (during JD48149-JD48345 and JD48905-JD49135). This is not surprising since the $\mathrm{H} \beta$ light curves of these three cases are sparsely sampled and suffer the most acute systematic errors. Nevertheless, the calculated $\chi^{2}$ distribution is generally consistent with the expectation, indicating that our modeling reasonably reproduces the emission-line data.

Figure 6 plots the distribution of the non-linearity parameter $\gamma$. Although the mean value of $\gamma$ is close to 0 , several objects exhibit prominent deviations. Indeed, it is common for the variability amplitude of the emission lines to exceed those of the continuum (Meusinger et al. 2011 and references therein). This can be simply verified by inspection of the "excess variance" values, which are usually provided in the literature. The non-linear response of $\mathrm{H} \beta$ might be ascribed to the following reasons: (1) The unobservable ionizing UV continuum is not linearly correlated with optical continuum (i.e., the shape of the spectral energy distribution is changing along with the continuum variation). This is plausible in light of the fact that, according to the standard accretion disk theory (Shakura \& Sunyaev 1973), the UV and optical emission mainly comes from different regions. (2) The portion of the BLR emitting $\mathrm{H} \beta$ may be (partially) optically thick so that the line intensity depends on the ionization parameter (e.g., Netzer 1990). However, in such a situation, $\mathrm{H} \beta$ emission may be anisotropic. We do not consider this effect in the present modeling. As mentioned above, RM observations of NGC 5548 tend to support the first explanation (Peterson et al. 2002; Bentz et al. 2007), although the second one cannot be excluded.

\subsection{Broad-line Region Geometry}

Figure 7 compares the $\mathrm{H} \beta$ BLR sizes derived from the present approach with those from the traditional analysis via cross-correlation functions (CCFs) between the continuum and the emission lines. We use the homogeneous compilation of CCF-based results from Bentz et al. (2013). Except for three objects PG 1411+442, NGC 5548 (JD54180-JD54332), and NGC 3783, the BLRs sizes derived using our approach are all systematically larger. Quantitatively, our derived BLR 

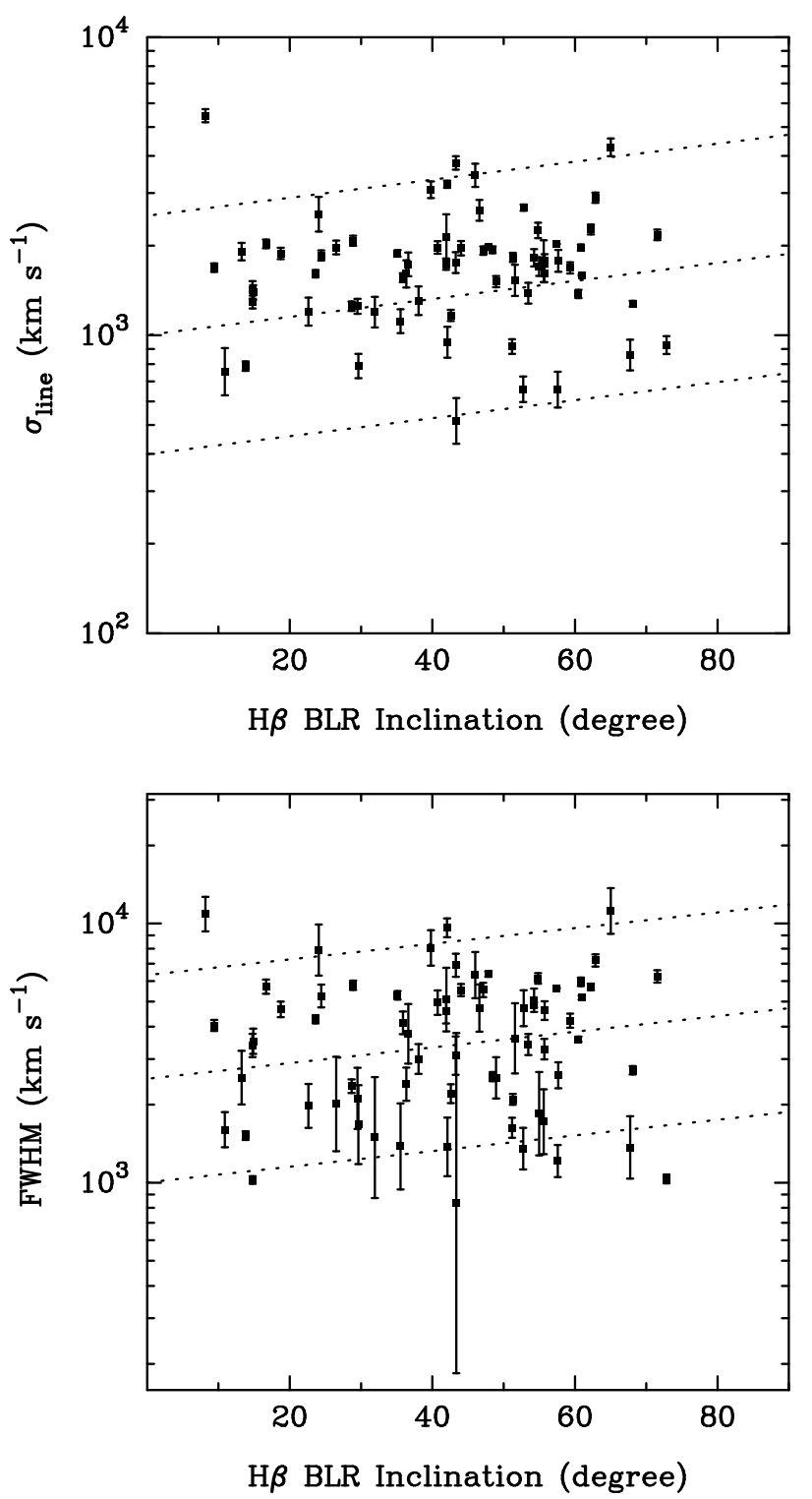

FIG. 8.- Dependence of $\mathrm{H} \beta$ (top) line dispersion and (bottom) FWHM measured from rms spectra with inclination angle. The uncertainties for the inclination angles are typically $20^{\circ}-30^{\circ}$ and are not shown for clarity. To guide the eye, the dotted lines plot the relation $V_{\mathrm{obs}}=V_{\mathrm{Kep}}\left(\sin ^{2} \theta_{\mathrm{opn}}+\right.$ $\left.\sin ^{2} \theta_{\text {inc }}\right)^{1 / 2}$ with $\theta_{\text {opn }}=40^{\circ}$ and $V_{\text {Kep }}=10^{2.8}, 10^{3.2}$, and $10^{3.6} \mathrm{~km} \mathrm{~s}^{-1}$ in the top panel and $V_{\mathrm{Kep}}=10^{3.2}, 10^{3.6}$, and $10^{4.2} \mathrm{~km} \mathrm{~s}^{-1}$ in the bottom panel.

sizes are on average larger than CCF-based sizes by $\sim 20 \%$. This confirms previous suspicions that $\mathrm{CCF}$ analysis might underestimate the sizes of BLRs (Netzer 1990; Maoz et al. 1991; Welsh 1999).

As discussed by Maoz et al. (1991), CCF analysis tends to weigh the inner parts of the BLR more than the outer parts ${ }^{10}$. The derived time lags will be considerably biased when the transfer functions are broad and multimodal. It is straightforward to derive the relation between the $\mathrm{CCF}$ and the transfer

\footnotetext{
${ }^{10}$ However, this does not at all mean that the current black hole mass measurements based on the $R_{\mathrm{BLR}}-L$ relationship are problematic. Note that the virial factors are calibrated using the lag determinations from CCF analysis.
}

function:

$$
\begin{aligned}
\operatorname{CCF}(\tau) & =\int f_{c}(t) f_{l}(t+\tau) \mathrm{d} t \\
& =A \int f_{c}(t) \int f_{c}^{1+\gamma}\left(t+\tau-\tau^{\prime}\right) \Psi\left(\tau^{\prime}\right) \mathrm{d} \tau^{\prime} \mathrm{d} t \\
& =A \int \Psi\left(\tau^{\prime}\right) \int f_{c}(t) f_{c}^{1+\gamma}\left(t+\tau-\tau^{\prime}\right) \mathrm{d} \tau^{\prime} \mathrm{d} t .
\end{aligned}
$$

Regardless of the non-linearity parameter $\gamma$ for now, we obtain a concise form (e.g., Welsh 1999),

$$
\operatorname{CCF}(\tau)=\int \Psi\left(\tau^{\prime}\right) \mathrm{ACF}_{c}\left(\tau-\tau^{\prime}\right) \mathrm{d} \tau^{\prime},
$$

where $\mathrm{ACF}_{c}$ refers to the autocorrelation function of the continuum

$$
\operatorname{ACF}_{c}\left(\tau-\tau^{\prime}\right)=\int f_{c}(t) f_{c}\left(t+\tau-\tau^{\prime}\right) \mathrm{d} t
$$

Consider an idealized case: $\mathrm{ACF}_{c}$ is a delta function so that the $\mathrm{CCF}$ is exactly identical to the transfer function. Consequently, multiple peaks in the transfer function (e.g., of an inclined disk-like BLR) lead to ambiguity in determining the peak values of the CCF. To be more specific, a thick, inclined disk has two peaks in the transfer function, and the stronger one is located closer in than the weaker one (e.g., see Figure 3 ). Hence, using the peak value of the CCF to determine the BLR size in such a circumstance biases the estimate toward the inner radius. A realistic situation will be even far more complicated once we account for the finite-duration, irregular sampling and observational noise.

Regarding the three cases whose size estimates may have been biased too low, it is too early to draw firm conclusions for PG 1411+442 and NGC 3783 because their light curves are sparsely sampled and noisy. However, for NGC 5548 (JD54180-JD54332), inspection of the CCF given by Denney et al. (2010) shows that it is quite broad, despite the high quality of the data set (see their Figure 3). This leads to a correspondingly broad transfer function (Figure 3), such that the CCF measurement can easily be skewed to incorrect values. Interestingly, the centroid value (12.4 days) of the $\mathrm{CCF}$ is twice as larger as the peak value (6.1 days).

In Figure 8, we show the inclination dependence of the $\mathrm{H} \beta$ line widths measured from rms spectra as line dispersion and FWHM. The error bars for the inclination angles are typically $20^{\circ}-30^{\circ}$ and are not plotted for clarity. To guide the eye, we superpose the relation described by Equation (20) with $\theta_{\text {opn }}=40^{\circ}$ and $V_{\text {Kep }}=10^{2.8}, 10^{3.2}$, and $10^{3.6} \mathrm{~km} \mathrm{~s}^{-1}$ in the top panel and $V_{\text {Kep }}=10^{3.2}, 10^{3.6}$, and $10^{4.2} \mathrm{~km} \mathrm{~s}^{-1}$ in the bottom panel. The relationship between line width and inclination angle has quite a large scatter because of the wide range of opening angles and $V_{\mathrm{Kep}}$, which depends on the central black hole mass and the BLR size. Nevertheless, it does seem that line width does increase slightly with inclination angle, in qualitative agreement with Equation (20), suggesting that the derived inclination angles are probably meaningful.

\subsection{Notes on Two Individual Objects}

We here demonstrate the ability of the present Bayesian approach to properly derive the BLR sizes for Mrk 142 and PG $2130+099$, both of which were previously considered to have unreliable lag determinations from $\mathrm{CCF}$ analysis because they 


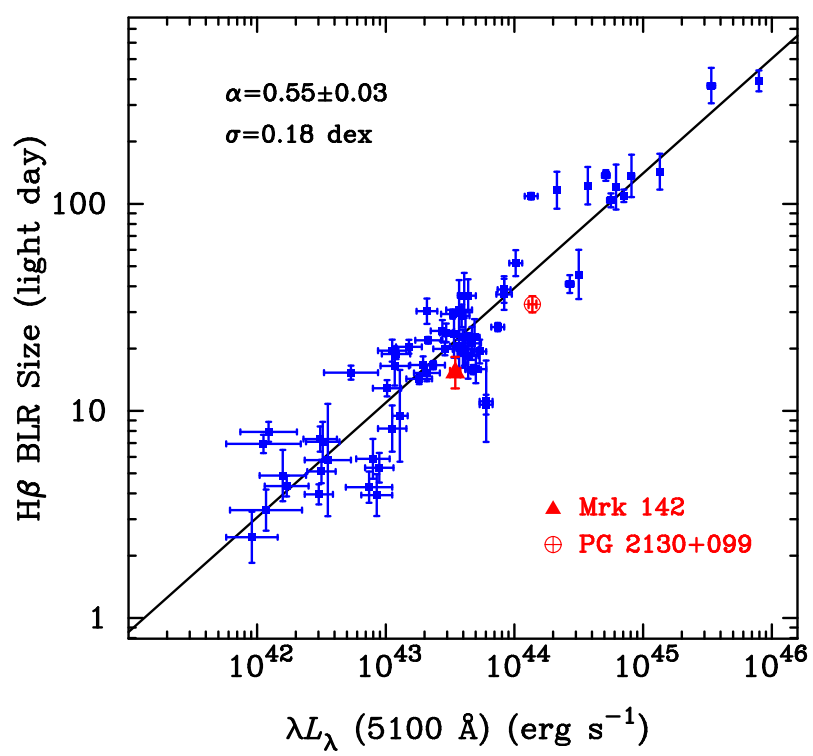

FIG. 9.- Relation between $\mathrm{H} \beta$ BLR size and $5100 \AA$ A luminosity. Solid line is the best linear fit.

were significant outliers in the $R_{\mathrm{BLR}}-L$ relation (Bentz et al. 2009b; Grier et al. 2012).

Mrk 142. This object was monitored by the LAMP project with a duration of $\sim 100$ days (Bentz et al. 2009b; Walsh et al. 2009). The data for the $\mathrm{H} \beta$ line were suspected to suffer from large systematic errors, probably due to FeII blending with [OIII], which was used to calibrate the $\mathrm{H} \beta$ fluxes (Bentz et al. $2009 \mathrm{~b})$. The CCF analysis by Bentz et al. (2009b) yielded an $\mathrm{H} \beta$ time lag of 2.87 days with respect to the $V$-band continuum, making Mrk 142 deviate strongly from the $R_{\mathrm{BLR}}-L$ relationship (Bentz et al. 2013). However, our modeling gives a lag of $15.3 \pm 2.7$ days, a factor of six larger than reported by Bentz et al. (2009b). The BLR size for Mrk 142 derived here is remarkably consistent with the $R_{\mathrm{BLR}}-L$ relationship.

$P G 2130+099$. This object was first monitored by Kaspi et al. (2000), who measured an $\mathrm{H} \beta$ time lag of 188 days, with a large uncertainty. Inspection of the $\mathrm{H} \beta$ light curve of Kaspi et al. (see their Figure 3) shows that a seasonal gap of a period of $\sim 200$ days may dominate the CCF and alias the lag determination. Later studies by Grier et al. (2012) with fairly wellsampled data determined a lag of 12.8 days, which falls well below the $R_{\mathrm{BLR}}-L$ relation (see also discussion in Bentz et al. 2013). However, a subsequent comprehensive investigation of the same data set by Grier et al. (2013b) using the maximum entropy technique found that the transfer function (or delay map) contains two peaks. The stronger one is located around 12.5 days and the weaker one at 31 days; therefore, the possibility of a lag of $\sim 31$ days cannot be excluded.

In our analysis, a thin disk model can reproduce the $\mathrm{H} \beta$ line fluxes with $\chi^{2} / \mathrm{dof}=1.19$. The inclination and opening angle are fairly well recovered: $\theta_{\text {inc }}=51^{\circ} \pm 6^{\circ}$ and $\theta_{\text {opn }}<18^{\circ}$. There are two peaks in the distribution of BLR size, located at $\sim 21$ and 33 days; the latter peak has significantly higher probability. On the other hand, it is also possible that PG 2130+099 undergoes secular variations. After applying our detrending procedure, we find a BLR size of $21.0 \pm 4.0$ with a $\chi^{2} / \mathrm{dof}=1.31$ (see online Figure 10). We adopt a BLR size of $32.7 \pm 3.0$ days, which places PG 2130+099 much closer to the $R_{\mathrm{BLR}}-L$ relationship.

\section{IMPLICATIONS FOR THE $R_{\mathrm{BLR}}-L$ RELATION}

The relationship between $\mathrm{H} \beta$ BLR size and $5100 \AA$ luminosity is plotted in Figure 9. As in Kaspi et al. (2005), we use the fitting routine FITEXY (Press et al. 1992) to include an intrinsic scatter, following the prescription of Tremaine et al. (2002). Specifically, we add in quadrature the uncertainty of $R_{\mathrm{BLR}}$ until we obtain a reduced $\chi^{2}$ of unity. The best fit gives

$$
\log \left(\frac{R_{\mathrm{BLR}}}{\mathrm{ld}}\right)=K+\alpha \log \left[\frac{\lambda L_{\lambda}(5100 \AA)}{10^{44} \mathrm{erg} \mathrm{s}^{-1}}\right],
$$

with $K=1.59 \pm 0.02$ and $\alpha=0.55 \pm 0.03$. The scatter of the fit is $0.18 \mathrm{dex}$, comparable to the 0.19 dex reported by Bentz et al. (2013). If our current analysis correctly models the geometry of the BLR, our derived $R_{\mathrm{BLR}}-L$ relation should account for the effect of inclination. That our inferred intrinsic scatter for the $R_{\mathrm{BLR}}-L$ relation is comparable to that of previous studies that did not include the inclination effect seems to imply that the scatter is dominated by other factors.

Intensive studies on NGC 5548 suggest that the slope of the $R_{\mathrm{BLR}}-L$ relation for individual objects may depart significant from the average slope. Using the 13-year optical and UV monitoring data of NGC 5548, Peterson et al. (2002) found that the $\mathrm{H} \beta$ lag correlates with UV flux as $\tau \propto F_{\mathrm{UV}}^{0.53}$, but, surprisingly, that it correlates almost linearly with the optical flux, $\tau \propto F_{\mathrm{opt}}^{0.93}$. If this behavior also holds for other RM objects, it should contribute a major source of scatter to the $R_{\mathrm{BLR}}-L$ relation. On the other hand, from a theoretical point of view, the shape of the emergent spectrum from the accretion disk depends on black hole mass, accretion rate, and as well as black hole spin. As the RM sample spans several orders of magnitude in luminosity, the correlation between the optical and ionizing UV luminosity should be quite diverse and hence must be a key source of scatter for the $R_{\mathrm{BLR}}-L$ relation. Other factors, such as Eddington ratio, may also contribute.

The best-fit slope of $\alpha=0.55 \pm 0.03$ is close to the value of $\alpha=0.53 \pm 0.03$ reported by Bentz et al. (2013), but marginally different from the expectation of 0.5 based on simple photoionization arguments. This is not surprising in light of the use of the optical continuum instead of the ionizing UV continuum and the non-linear response of the line emission, which implies that the shape of the incident optical-UV continuum might be changing with reverberation variations or, alternatively, that the BLR is optically thick to $\mathrm{H} \beta$. Both of these two possibilities violate the simplistic assumptions of the photoionization argument. As mentioned above, longterm monitoring of NGC 5548 provides evidence that the shape of the continuum does change (Peterson et al. 2002; see also Bentz et al. 2007).

\section{DISCUSSION}

We have demonstrated the fidelity of our approach for diagnosing the structure of the BLR, in particular in its ability to reproduce the light curves of emission line fluxes. Compared with the original framework developed by Pancoast et al. (2011), we explicitly include the non-linear response of the line emission to the continuum and detrending of the light curves to remove long-term secular variability sometimes seen in both the emission lines and continuum. The transfer function is self-consistently calculated in the present approach, obviating the need assume it, as in $\mathrm{Zu}$ et al. (2011). However, we do need to specify a general BLR model in ad- 
vance for the implementation of the Bayesian analysis. Thus, we cannot claim that the BLR model is unique. In the future, we can circumvent this limitation by model selection when the observations are of sufficient quality to justify construction of more refined BLR models.

Previous studies that have succeeded in recovering velocitydelay maps suggest that multiple components coexist in the BLR, including disks, winds, and inflows/outflows (e.g., Bentz et al. 2010; Grier et al. 2013b; see also Peterson 2013 for a review). This does not invalidate the present modeling, which makes use of only the $\mathrm{H} \beta$ light curves. Only with velocity-resolved mapping data can these different kinematics be distinguished. Without any additional information on the preferred geometry of the BLR, we proceed with the assumption that the BLR has a flexible disk geometry.

Future inclusion of emission line profiles will definitely provide more stringent constraints on the BLR geometry in general, and on the inclination angle in particular (see the works of Brewer et al. 2011 and Pancoast et al. 2012). It will then be instructive to try to compare the inclination of the BLR with indicators of the orientation of the accretion disk (e.g., Nishiura et al. 1998; Wu \& Han 2001; Jarvis \& McLure 2006; Runnoe et al. 2013). This will allow us test models for the formation and evolution of the BLR (see Wang et al. 2011, 2012 and references therein). The most promising indicator of the orientation of the accretion disk is the relativistically broadened $\mathrm{Fe} \mathrm{K} \alpha$ emission line, which is believed to originate from the inner region of the accretion disk and hence should trace the overall inclination of the disk (e.g., Patrick et al. 2012; Walton et al. 2013 and references therein). The heuristic work by Nishiura et al. (1998) correlated the disk inclination, derived from early rudimentary modeling of broad $\mathrm{Fe} \mathrm{K} \alpha$, with emission-line widths and concluded that the BLR may arise from the outer parts of a warped accretion disk.

The present approach might fail to reproduce the correct BLR size in cases where the light curve is poorly sampled or suffers from severe seasonal aliasing (e.g., 3C 120 in Peterson et al. 1998). However, these cases can be easily singled out through simple visual inspection. Supplying Equation (17) with more sophisticated priors or using sections of the light curve with relatively better sampling rates helps to eliminate seasonal aliasing. In addition, as suggested by $\mathrm{Zu}$ et al. (2011), using multiple emission lines can further alleviate sampling problems and seasonal aliasing. For this purpose, one needs to simultaneously fit the continuum and multiple emission lines ( $\mathrm{Zu}$ et al. 2011), and one needs to treat the entire continuum as unknown but to be inferred from the data (Pancoast et al. 2011). This will be computationally challenging and is unfeasible for analyzing a large sample. We are currently developing a parallelization of our present approach using a supercomputer cluster to address these issues.

It is worth pointing out that the size and structure of BLRs potentially vary with emission lines. RM studies of various emission lines see evidence for stratification of the BLR; namely, low-ionization lines (such as $\mathrm{H} \beta$ and $\mathrm{C}$ III) respond with longer time delays than higher ionization lines (such as He II and C IV; e.g., Clavel et al. 1991; Dietrich \& Kollatschny 1995; Kollatschny 2003). This indicates that BLRs are obviously much more complicated than our simple modeling. An extension of the present approach by self-consistently including multiple emission lines will shed light on the global structures of BLRs.

Apart from the above points, there are several other aspects for future improvement.
1. For simplicity, we assume that the continuum fluxes are isotropic and decline as the inverse square of the distance of clouds from the central ionizing source. More sophisticated cases are beyond the goal of this work, but are worth studying in the future with high-fidelity data.

2. Incorporate realistic and physical ionization calculations (e.g., using the well-developed package CLOUDY; Ferland et al. 1998) to take into account the effects of optical depth and probe the physical environment of the BLR (Chiang \& Murray 1996; Ferland et al. 2009; see also Horne et al. 2003).

3. Include the influence of radiation pressure on the kinematics of the BLR clouds; radiation pressure is believed to lead to underestimates of the black hole mass for objects with high Eddington ratios (Marconi et al. 2008; Netzer 2009).

4. Construct physical models for inflows and outflows, plausible components inferred in a number of previous studies (e.g., Kollatschny \& Dietrich 1996; Gaskell \& Goosmann 2013).

\section{CONCLUSIONS}

Motivated by recent advances in BLR modeling and statistical description of AGN variability, we carry out a systematic study of BLR structure using all the RM data with $\mathrm{H} \beta$ monitoring available in the literature. We improve on previous efforts by incorporating the non-linearity of the line response to the continuum and by detrending the light curves for secular variability. Although a general disk geometry is assumed in this initial work, the flexibility of the Bayesian approach readily allows us in the future to account for more complicated BLR structures and physical processes. Our main results are as follows.

1. The damped random walk model can explain the variability of the optical continuum for all the RM objects, confirming the results of previous studies (e.g., Kelly et al. 2009; $\mathrm{Zu}$ et al. 2013). The advantage with RM sample is that the black hole mass can be simply estimated, thus permitting the physical origin of the variability to be investigated by linking it to the properties of the accretion disk (e.g., Eddington ratio, orbital motion, thermal processes).

2. The observed $\mathrm{H} \beta$ light curves can be fairly well reproduced by a general geometry for the BLR that accounts for disks, rings, shells, and spheres. This indicates that the structure of the BLR for $\mathrm{H} \beta$ emission line is mainly disk-like.

3. The $\mathrm{H} \beta$ BLR sizes determined here through our Bayesian method are systematically larger by $\sim 20 \%$ compared to those derived from the traditional crosscorrelation analysis. This discrepancy plausibly arises from the fact that the cross-correlation method biases is biased toward the inner parts of the BLR (Netzer 1990; Maoz et al. 1991; Welsh 1999).

4. We redetermine the $R_{\mathrm{BLR}}-L$ relationship and find a slope of $0.55 \pm 0.03$ and an intrinsic scatter of $0.18 \mathrm{dex}$. Since the derived BLR sizes have already taken into account the inclination effect, the remaining scatter must 
arise from other factors, including variation in the shape of the continuum and diverse Eddington ratios.

5. In present framework, we find that the non-linear response of the $\mathrm{H} \beta$ line emission to the continuum is required to better reproduce the observed line fluxes (see Figure 6). This seems reasonable, in light of the fact that the variation amplitude of the line emission sometimes exceeds that of the continuum. The non-linearity may be ascribed to the non-linear correlation between the optical and ionizing UV continuum (Peterson et al. 2002) or to the existence of (partially) optically thick BLR clouds.

6. We demonstrate the capability of the present approach to recover appropriate BLR sizes when the traditional cross-correlation analysis fails. The newly obtained BLR sizes for Mrk 142 and PG 2130+099, previously reported to be major outliers of the $R_{\mathrm{BLR}}-L$ relationship (Bentz et al. 2013), shows remarkable consistency with the anticipated values (see Figure 9).

We thank the members of IHEP AGN group for discussions. This research is supported by NSFC-11133006, 11173023, 11233003 , and 11303026, a 973 project (2009CB824800), and the China-Israel NSFC-ISF 11361140347. The work of L.C.H. is supported by the Kavli Foundation, Peking University, and the Carnegie Institution for Science.

\section{APPENDIX}

\section{EQUATIONS FOR THE CONTINUUM RECONSTRUCTION}

We derive the equations for the continuum reconstruction described in Section 2.1. Assuming that both the signal $s$ and the noise $n$ have a Gaussian distribution with covariance matrices $S=\left\langle s s^{T}\right\rangle$ and $N=\left\langle n n^{T}\right\rangle$, respectively, their probability distributions are

$$
P(s) \propto|S|^{-1 / 2} \exp \left(-\frac{s^{T} S^{-1} s}{2}\right),
$$

and

$$
P(n) \propto|\boldsymbol{N}|^{-1 / 2} \exp \left(-\frac{n^{T} N^{-1} n}{2}\right),
$$

where the angle bracket denotes statistical ensemble average and the superscript " $T$ " denotes the transposition of vectors or matrices. We further assume that $s$ and $n$ are uncorrelated, so that the joint probability $P(s, n)=P(s) P(\boldsymbol{n})$. Now we calculate the probability for the observations $\boldsymbol{y}=\boldsymbol{s}+\boldsymbol{n}+\boldsymbol{E} q$ as (Rybicki \& Press 1992)

$$
\begin{aligned}
P\left(\boldsymbol{y} \mid q, \sigma_{\mathrm{d}}, \tau_{\mathrm{d}}\right) & =\int P(\boldsymbol{s}, \boldsymbol{n}) \delta[\boldsymbol{y}-(\boldsymbol{s}+\boldsymbol{n}+\boldsymbol{E} q)] \mathrm{d}^{m} \boldsymbol{s} \mathrm{d}^{m} \boldsymbol{n} \\
& =\int P(\boldsymbol{s}) P(\boldsymbol{n}=\boldsymbol{y}-\boldsymbol{E} q-\boldsymbol{s}) \mathrm{d}^{m} \boldsymbol{s} \\
& \propto \exp \left[-\frac{1}{2}(\boldsymbol{y}-\boldsymbol{E} q)^{T}(\boldsymbol{S}+\boldsymbol{N})^{-1}(\boldsymbol{y}-\boldsymbol{E} q)\right] \int \exp \left[-\frac{1}{2}(\boldsymbol{s}-\hat{\boldsymbol{s}})^{T}\left(\boldsymbol{S}^{-1}+\boldsymbol{N}^{-1}\right)(\boldsymbol{s}-\hat{\boldsymbol{s}})\right] \mathrm{d}^{m} \boldsymbol{s},
\end{aligned}
$$

where $\hat{s}=S(S+N)^{-1}(y-E q)$ and the equalities $\left(S^{-1}+N^{-1}\right)^{-1}=S(S+N)^{-1} N=N(S+N)^{-1} S$ are used. Substituting the variable of integration with $(s-\hat{s})$ and manipulating a simple normalization yields

$$
P\left(\boldsymbol{y} \mid q, \sigma_{\mathrm{d}}, \tau_{\mathrm{d}}\right)=\frac{1}{\sqrt{(2 \pi)^{m}|\boldsymbol{C}|}} \exp \left[-\frac{1}{2}(\boldsymbol{y}-\boldsymbol{E} q)^{T} \boldsymbol{C}^{-1}(\boldsymbol{y}-\boldsymbol{E} q)\right],
$$

where $C \equiv S+N$. We marginalize the parameter $q$ by "completing the square"

$$
(\boldsymbol{y}-\boldsymbol{E} q)^{T} \boldsymbol{C}^{-1}(\boldsymbol{y}-\boldsymbol{E} q)=(\boldsymbol{y}-\boldsymbol{E} \hat{q})^{T} \boldsymbol{C}^{-1}(\boldsymbol{y}-\boldsymbol{E} \hat{q})+(q-\hat{q}) \boldsymbol{E}^{T} \boldsymbol{C}^{-1} \boldsymbol{E}(q-\hat{q}),
$$

where

$$
\hat{q}=\frac{\boldsymbol{E}^{T} \boldsymbol{C}^{-1} \boldsymbol{y}}{\boldsymbol{E}^{T} \boldsymbol{C}^{-1} \boldsymbol{E}} .
$$

This formula is the generalized "inverse-variance weighted mean" that accounts for the correlation between data points in a way that a data value highly correlated with other data value gets a small weight (Rybicki \& Press 1992). As a result, by assuming, as usual, that $P(q)$ is constant, we arrive at

$$
P\left(\boldsymbol{y} \mid \sigma_{\mathrm{d}}, \tau_{\mathrm{d}}\right)=\int P\left(\boldsymbol{y} \mid q, \sigma_{\mathrm{d}}, \tau_{\mathrm{d}}\right) P(q) \mathrm{d} q=\frac{1}{\sqrt{(2 \pi)^{m}|\boldsymbol{C}|}} \exp \left[-\frac{1}{2}(\boldsymbol{y}-\boldsymbol{E} \hat{q})^{T} \boldsymbol{C}^{-1}(\boldsymbol{y}-\boldsymbol{E} \hat{q})\right] .
$$

From above manipulation, one readily finds the best estimate of $y$ :

$$
\hat{\boldsymbol{y}}=\hat{s}+\boldsymbol{E} \hat{q}=\boldsymbol{S} \boldsymbol{C}^{-1}(\boldsymbol{y}-\boldsymbol{E} \hat{q})+\boldsymbol{E} \hat{q} .
$$



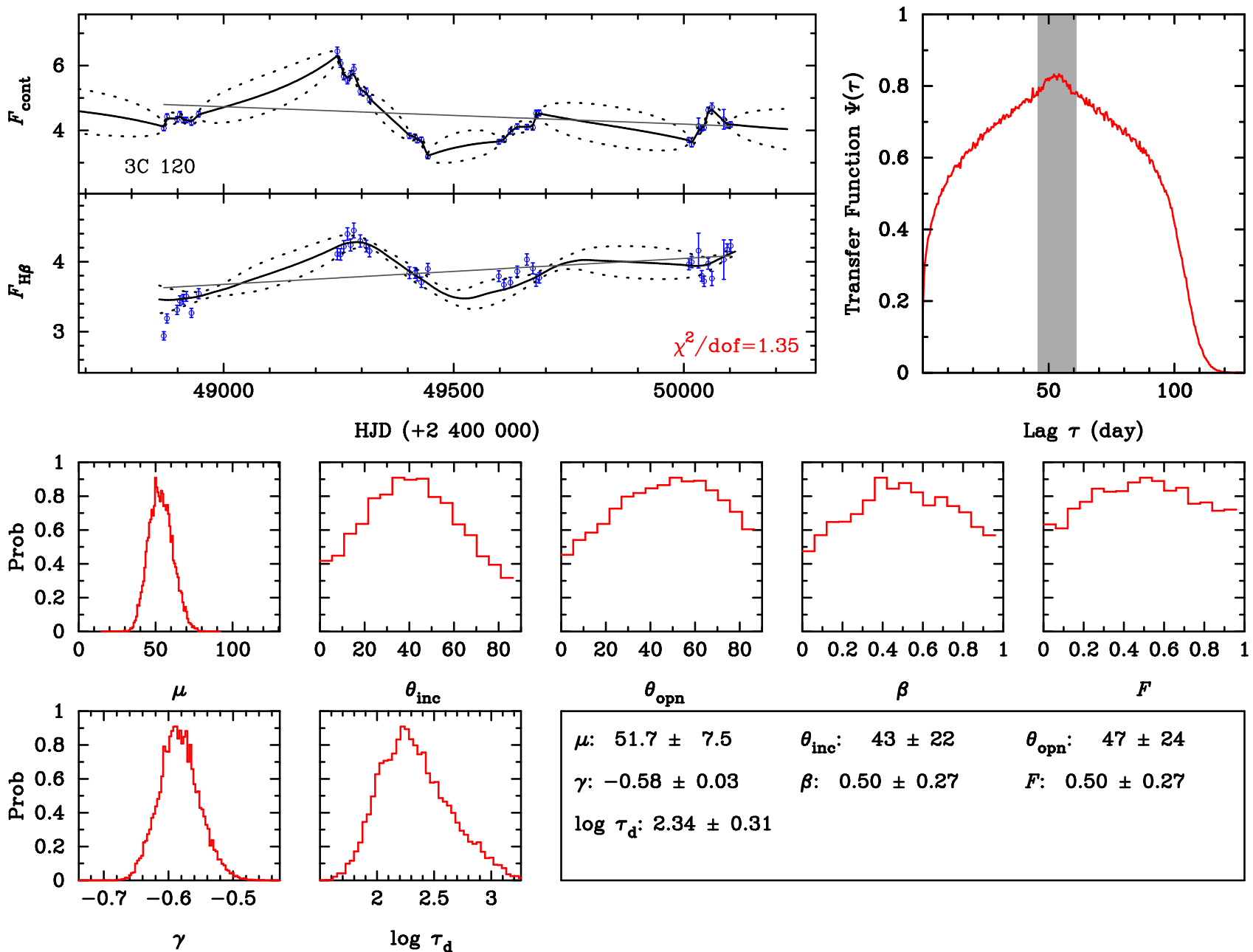

FIG. 10._ Continuum reconstructions and BLR modelings. (Top left) Data points with error bars are the observed light curves. Thick solid lines show the reconstructed continuum at $5100 \AA$ and the recovered $\mathrm{H} \beta$ emission; dashed lines represent the uncertainties. If a detrending is initiated, the gray lines show the fits for the detrending applied to remove the secular variations. (Top right) Transfer function $\Psi(\tau)$ (red curve, in arbitrary units) derived from the best-fit BLR model. The vertical shaded area represents the recovered BLR size and its uncertainty in observer's frame. (Bottom) Distributions for the parameters of mean radius $\mu$ (light day), inclination angle $\theta_{\text {inc }}$ (degree), opening angle $\theta_{\mathrm{opn}}$ (degree), $\beta, F$, non-linearity $\gamma$, and typical damping timescale $\tau_{\mathrm{d}}$ (day). Their statistical values are summarized in bottom right panel. Note that here $\mu$ and $\tau_{\mathrm{d}}$ are given in the rest-frame.

(The complete figure set (71 images) and color version are available in the online journal.)

The mean square residual of the estimate is calculated by

$$
\begin{aligned}
\left\langle[\hat{\boldsymbol{y}}-(s-\boldsymbol{E} q)]^{2}\right\rangle & =\left\langle\left[\boldsymbol{S} \boldsymbol{C}^{-1}(\boldsymbol{y}-\boldsymbol{E} \hat{q})+\boldsymbol{E} \hat{q}-s\right]\left[\boldsymbol{S} \boldsymbol{C}^{-1}(\boldsymbol{y}-\boldsymbol{E} \hat{q})+\boldsymbol{E} \hat{q}-s\right]^{T}\right\rangle \\
& =\boldsymbol{S}-\boldsymbol{S}^{T} \boldsymbol{C}^{-1} \boldsymbol{S}+\frac{\left(\boldsymbol{S} \boldsymbol{C}^{-1} \boldsymbol{E}-\boldsymbol{E}\right)\left(\boldsymbol{S} \boldsymbol{C}^{-1} \boldsymbol{E}-\boldsymbol{E}\right)^{T}}{\boldsymbol{E}^{T} \boldsymbol{C}^{-1} \boldsymbol{E}},
\end{aligned}
$$

where the terms invoking $q$ are eliminated for ensemble averages.

\section{ONLINE FIGURES}

In the online version of Figure 10, we plot the reconstructions of the continuum light curves, the fits for the $\mathrm{H} \beta$ light curves, and the recovered parameter distributions of the BLR for all the objects in the sample, in the order as they appear in Table 1.

\section{REFERENCES}

Bentz, M. C., Denney, K. D., Cackett, E. M., et al. 2006, ApJ, 651, 775

Bentz, M. C., Denney, K. D., Cackett, E. M., et al. 2007, ApJ, 662, 205

Bentz, M. C., Denney, K. D., Grier, C. J., et al. 2013, ApJ, 767, 149

Bentz, M. C., Horne, K., Barth, A. J., et al. 2010, ApJL, 720, 46

Bentz, M. C., Peterson, B. M., Netzer, H., Pogge, R. W., \& Vestergaard, M. 2009a, ApJ, 697, 160

Bentz, M. C., Walsh, J. L., Barth, A. J., et al. 2009b, ApJ, 705, 199

Blandford, R. D., \& McKee, C. F. 1982, ApJ, 255, 419
Brewer, B. J., Treu, T., Pancoast, A., et al. 2011, ApJL, 733, 33

Chiang, J., \& Murray, N. 1996, ApJ, 466, 704

Clavel, J., Reichert, G. A., Alloin, D., et al. 1991, ApJ, 366, 64

Collin, S., Kawaguchi, T., Peterson, B. M., \& Vestergaard, M. 2006, A\&A, 456, 75

Crenshaw, D. M., Kraemer, S. B., Bruhweiler, F. C., \& Ruiz, J. R. 2001, ApJ, 555, 633

Davies, R. I., Thomas, J., Genzel, R., et al. 2006, ApJ, 646, 754 
Decarli, R., Labita, M., Treves, A., \& Falomo, R. 2008, MNRAS, 387, 1237 Denney, K. D., Bentz, M. C., Peterson, B. M., et al. 2006, ApJ, 653, 152 Denney, K. D., Peterson, B. M., Pogge, R. W., et al. 2010, ApJ, 721, 715 Denney, K. D., Watson, L. C., Peterson, B. M., et al. 2009, ApJ, 702, 1353 Dexter, J., \& Agol, E. 2011, ApJL, 727, 24

Dietrich, M., \& Kollatschny, W. 1995, A\&A, 303, 405

Dietrich, M., Peterson, B. M., Albrecht, P., et al. 1998, ApJS, 115, 185

Dietrich, M., Peterson, B. M., Grier, C. J., et al. 2012, ApJ, 757, 53

Eracleous, M., \& Halpern, J. P. 2003, ApJ, 599, 886

Eracleous, M., Lewis, K. T., \& Flohic, H. M. L. G. 2009, New Astron. Rev., 53,133

Ferland, G. J., Hu, C., Wang, J.-M., et al. 2009, ApJL, 707, 82

Ferland, G. J., Korista, K. T., Verner, D. A., et al. 1998, PASP, 110, 761

Gaskell, C. M., \& Goosmann, R. W. 2013, ApJ, 769, 30

Gaskell, C. M. \& Sparke, L. S. 1986, ApJ, 305, 175

Gezari, S., Halpern, J. P., \& Eracleous, M. 2007, ApJS, 169, 167

Goad, M. R., Korista, K. T., \& Ruff, A. J. 2012, MNRAS, 426, 3086

Graham, A. W., Onken, C. A., Athanassoula, E., \& Combes, F. 2011, MNRAS, 412, 2211

Greene, J. E., \& Ho, L. C. 2007, ApJ, 667, 131

Grier, C. J., Martini, P., Watson, L. C., et al. 2013a, ApJ, 773, 90

Grier, C. J., Peterson, B. M., Horne, K., et al. 2013b, ApJ, 764, 47

Grier, C. J., Peterson, B. M., Pogge, R. W., et al. 2012, ApJ, 755, 60

Gültekin, K., Richstone, D. O., Gebhardt, K., et al. 2009, ApJ, 698, 198

Hicks, E. K. S., \& Malkan, M. A. 2008, ApJS, 174, 31

Ho, L. C. 2008, ARA\&A, 46, 475

Horne, K. 1994, in ASP Conf. Ser. 69, Reverberation Mapping of the Broad

Line Region in Active Galactic Nuclei, ed. P. M. Gondhalekar, K. Horne,

\& B. M. Peterson (San Francisco, CA: ASP), 23

Horne, K., Korista, K. T., \& Goad, M. R. 2003, MNRAS, 339, 367

Horne, K., Welsh, W. F., \& Peterson, B. M. 1991, ApJL, 367, 5

Jarvis, M. J., \& McLure, R. J. 2006, MNRAS, 369, 182

Kaspi, S., Maoz, D., Netzer, H., et al. 1996, ApJ, 470, 336

Kaspi, S., Maoz, D., Netzer, H., et al. 2005, ApJ, 629, 61

Kaspi, S., Smith, P. S., Netzer, H., et al. 2000, ApJ, 533, 631

Kelly, B. C., Bechtold, J., \& Siemiginowska, A. 2009, ApJ, 698, 895

Kelly, B. C., Sobolewska, M., \& Siemiginowska, A. 2011, ApJ, 730, 52

Kollatschny, W. 2003, A\&A, 407, 461

Kollatschny, W., \& Dietrich, M. 1996, A\&A, 314, 43

Kormendy, J., \& Ho, L. C. 2013, ARA\&A, 51, 511

Kozłowski, S., Kochanek, C. S., Udalski, A., et al. 2010, ApJ, 708, 927

Krolik, J. H., \& Done, C. 1995, ApJ, 440, 166

Lewis, K. T., Eracleous, M., \& Storchi-Bergmann, T. 2010, ApJS, 187, 416

Li, Y.-R., Ho, L. C., \& Wang, J.-M. 2011, ApJ, 742, 33

Li, Y.-R., Wang, J.-M., \& Ho, L. C. 2012, ApJ, 749, 187

Liu, J. S. 2001, Monte Carlo Strategies in Scientific Computing (New York: Springer)

MacLeod, C. L., Brooks, K., Ivezić, Ž., et al. 2011, ApJ, 728, 26

MacLeod, C. L., Ivezić, Ž., Kochanek, C. S., et al. 2010, ApJ, 721, 1014

Maoz, D., Netzer, H., Mazeh, T., et al. 1991, ApJ, 367, 493

Marconi, A., Axon, D. J., Maiolino, R., et al. 2008, ApJ, 678, 693

Marconi, A., Risaliti, G., Gilli, R., et al. 2004, MNRAS, 351, 169

Meusinger, H., Hinze, A., \& de Hoon, A. 2011, A\&A, 525, A37

Netzer, H. 1990, in Active Galactic Nuclei, ed. R. D. Blandford, H. Netzer,

L. Woltjer, T. J.-L. Courvoisier, \& M. Mayor (Berlin: Springer), 57

Netzer, H. 2009, ApJ, 695, 793

Netzer, H., Elitzur, M., \& Ferland, G. J. 1985, ApJ, 299, 752

Nishiura, S., Murayama, T., \& Taniguchi, Y. 1998, PASJ, 50, 31
Onken, C. A., Ferrarese, L., Merritt, D., et al. 2004, ApJ, 615, 645

Onken, C. A., \& Peterson, B. M. 2002, ApJ, 572, 746

Onken, C. A., Valluri, M., Peterson, B. M., et al. 2007, ApJ, 670, 105

Pancoast, A., Brewer, B. J., \& Treu, T. 2011, ApJ, 730, 139

Pancoast, A., Brewer, B. J., Treu, T., et al. 2012, ApJ, 754, 49

Park, D., Kelly, B., Woo, J.-H., \& Treu, T. 2013, ApJS, 203, 6

Patrick, A. R., Reeves, J. N., Porquet, D., et al. 2012, ApJ, 426, 2522

Peterson, B. M. 1993, PASP, 105, 247

Peterson, B. M. 2013, Space Sci. Rev. in press

Peterson, B. M., Berlind, P., Bertram, R., et al. 2002, ApJ, 581, 197

Peterson, B. M., Ferrarese, L., Gilbert, K. M., et al. 2004, ApJ, 613, 682

Peterson, B. M., Wanders, I., Bertram, R., et al. 1998, ApJ, 501, 82

Pijpers, F. P., \& Wanders, I. 1994, MNRAS, 271, 183

Press, W. H., Teukolsky, S. A., Vetterling, W. T., \& Flannery, B. P. 1992,

Numerical Recipes in FORTRAN. The Art of Scientific Computing (2nd

ed.; Cambridge: Cambridge Univ. Press), 660

Robinson, A., \& Perez, E. 1990, MNRAS, 244, 138

Rodriguez-Pascual, P. M., Alloin, D., Clavel, J., et al. 1997, ApJS, 110, 9

Rokaki, E., Lawrence, A., Economou, F., \& Mastichiadis, A. 2003,

MNRAS, 340, 1298

Runnoe, J. C., Brotherton, M. S., Shang, Z., Wills, B. J., \& DiPompeo,

M. A. 2013, MNRAS, 429,135

Rybicki, G. B., \& Press, W. H. 1992, ApJ, 398, 169

Santos-Lleó, M., Chatzichristou, E., Mendes de Oliveira, C., et al. 1997,

ApJS, 112, 271

Santos-Lleó, M., Clavel, J., Schulz, B., et al. 2001, A\&A, 369, 57

Sergeev, S. G., Doroshenko, V. T., Dzyuba, S. A., et al. 2007, ApJ, 668, 708

Sergeev, S. G., Pronik, V. I., \& Sergeeva, E. A. 2000, A\&A, 356, 41

Shakura, N. I., \& Sunyaev, R. A. 1973, A\&A, 24, 337

Shapovalova, A. I., Doroshenko, V. T., Bochkarev, N. G., et al. 2004, A\&A, 422, 925

Shen, Y., Greene, J. E., Strauss, M. A., Richards, G. T., \& Schneider, D. P. 2008, ApJ, 680, 169

Sivia, D. \& Skilling, D. 2006, Data Analysis: A Bayesian Tutorial (New

York: Oxford Univ. Press), P. 6

Stirpe, G. M., Winge, C., Altieri, B., et al. 1994, ApJ, 425, 609

Strateva, I. V., Strauss, M. A., Hao, L., et al. 2003, AJ, 126, 1720

Tremaine, S., Gebhardt, K., Bender, R., et al. 2002, ApJ, 574, 740

Ulrich, M.-H., \& Horne, K. 1996, MNRAS, 283, 748

Vestergaard, M., \& Osmer, P. S. 2009, ApJ, 699, 800

Vestergaard, M. \& Peterson, B. M. 2006, ApJ, 641, 689

Walsh, J. L., Minezaki, T., Bentz, M. C., et al. 2009, ApJS, 185, 156

Walton, D. J., Nardini, E., Fabian, A. C., Gallo, L. C., \& Reis, R. C. 2013, MNRAS, 428, 2901

Wanders, I., Goad, M. R., Korista, K. T., et al. 1995, ApJL, 453, 87

Wanders, I., \& Peterson, B. M. 1996, ApJ, 466, 174

Wang, J.-M., Du, P., Baldwin, J. A., et al. 2012, ApJ, 746, 137

Wang, J.-M., Ge, J.-Q., Hu, C., et al. 2011, ApJ, 739, 3

Wang, J.-M., Hu, C., Li, Y.-R., et al. 2009, ApJL, 697, 141

Welsh, W. F. 1999, PASP, 111, 1347

Welsh, W. F., \& Horne, K. 1991, ApJ, 379, 586

Woo, J.-H., Treu, T., Barth, A. J., et al. 2010, ApJ, 716, 269

Wu, X.-B., \& Han, J. L. 2001, ApJL, 561, 59

Zu, Y., Kochanek, C. S., Kozłowski, S., \& Udalski, A. 2013, ApJ, 765, 106

Zu, Y., Kochanek, C. S., \& Peterson, B. M. 2011, ApJ, 735, 80

TABLE 1

OBJECT PROPERTIES

\begin{tabular}{lcccccc}
\hline \hline Object & Redshift & $\begin{array}{c}\text { Julian Dates } \\
(+2400000)\end{array}$ & $\begin{array}{c}\log \lambda L_{\lambda}(5100 \AA) \\
\left(\mathrm{erg} \mathrm{s}^{-1}\right)\end{array}$ & $\begin{array}{c}\text { FWHM } \\
\left(\mathrm{km} \mathrm{s}^{-1}\right)\end{array}$ & $\begin{array}{c}\sigma_{\text {line }} \\
\left(\mathrm{km} \mathrm{s}^{-1}\right)\end{array}$ & Reference \\
\hline 3C 120 & 0.03301 & $48869-50101$ & $44.01 \pm 0.05$ & $2205 \pm 185$ & $1166 \pm 50$ & 1 \\
$\ldots$ & $\ldots$ & $55430-55569$ & $43.87 \pm 0.05$ & $2539 \pm 466$ & $1514 \pm 65$ & 2 \\
3C 390.3 & 0.05610 & $49718-50012$ & $43.62 \pm 0.10$ & $9630 \pm 804$ & $3211 \pm 90$ & 3 \\
$\ldots$ & $\ldots$ & $53630-53713$ & $44.43 \pm 0.03$ & $10872 \pm 1670$ & $5455 \pm 278$ & 4 \\
Ark 120 & 0.03230 & $48149-48345$ & $43.92 \pm 0.06$ & $5536 \pm 297$ & $1959 \pm 109$ & 1 \\
$\ldots$ & $\ldots$ & $48870-49090$ & $43.57 \pm 0.10$ & $5284 \pm 203$ & $1884 \pm 48$ & 1 \\
Arp 151 & 0.02109 & $54506-54607$ & $42.48 \pm 0.11$ & $2357 \pm 142$ & $1252 \pm 46$ & 5,6 \\
Fairall 9 & 0.04702 & $50473-50665$ & $43.92 \pm 0.05$ & $6901 \pm 707$ & $3787 \pm 197$ & 7,8 \\
Mrk 79 & 0.02219 & $47838-48044$ & $43.57 \pm 0.07$ & $5086 \pm 1436$ & $2137 \pm 375$ & 1 \\
$\ldots$ & $\ldots$ & $48193-48393$ & $43.67 \pm 0.07$ & $4219 \pm 262$ & $1683 \pm 72$ & 1 \\
$\ldots$ & $\ldots$ & $48905-49135$ & $43.60 \pm 0.07$ & $5251 \pm 533$ & $1854 \pm 72$ & 1 \\
Mrk 110 & 0.03529 & $48954-49149$ & $43.62 \pm 0.04$ & $1494 \pm 802$ & $1196 \pm 141$ & 1 \\
$\ldots$ & $\ldots$ & $49752-49875$ & $43.69 \pm 0.04$ & $1381 \pm 528$ & $1115 \pm 103$ & 1 \\
$\ldots$ & $\ldots$ & $50011-50262$ & $43.47 \pm 0.05$ & $1521 \pm 59$ & $788 \pm 29$ & 1 \\
Mrk 142 & 0.04494 & $54506-54618$ & $43.54 \pm 0.04$ & $1368 \pm 379$ & $859 \pm 102$ & 5,6 \\
Mrk 202 & 0.02102 & $54505-54617$ & $42.20 \pm 0.18$ & $1354 \pm 250$ & $659 \pm 65$ & 5,6 \\
Mrk 279 & 0.03045 & $50095-50289$ & $43.64 \pm 0.08$ & $3385 \pm 349$ & $1420 \pm 96$ & 9 \\
Mrk 290 & 0.02958 & $54180-54321$ & $43.11 \pm 0.06$ & $4270 \pm 157$ & $1609 \pm 47$ & 10 \\
Mrk 335 & 0.02578 & $48156-49338$ & $43.70 \pm 0.06$ & $1629 \pm 145$ & $917 \pm 52$ & 1 \\
\hline & & & & & & \\
\hline
\end{tabular}


TABLE 1 - Continued

\begin{tabular}{|c|c|c|c|c|c|c|}
\hline Object & Redshift & $\begin{array}{l}\text { Julian Dates } \\
(+2400000)\end{array}$ & $\begin{array}{c}\log \lambda L_{\lambda}(5100 \AA) \\
\left(\operatorname{erg~s}^{-1}\right)\end{array}$ & $\begin{array}{l}\text { FWHM } \\
\left(\mathrm{km} \mathrm{s}^{-1}\right)\end{array}$ & $\begin{array}{c}\sigma_{\text {line }} \\
\left(\mathrm{km} \mathrm{s}^{-1}\right)\end{array}$ & Reference \\
\hline$\ldots$ & $\ldots$ & $49889-50118$ & $43.78 \pm 0.05$ & $1375 \pm 357$ & $948 \pm 113$ & 1 \\
\hline$\ldots$ & . & $55431-55569$ & $43.68 \pm 0.06$ & $1025 \pm 35$ & $1293 \pm 64$ & 2 \\
\hline Mrk 509 & 0.03440 & $47653-50374$ & $44.13 \pm 0.05$ & $2715 \pm 101$ & $1276 \pm 28$ & 1 \\
\hline Mrk 590 & 0.02638 & $48090-48323$ & $43.53 \pm 0.07$ & $1675 \pm 587$ & $789 \pm 74$ & 1 \\
\hline$\ldots$ & $\ldots$ & $48848-49048$ & $43.07 \pm 0.11$ & $2566 \pm 106$ & $1935 \pm 52$ & 1 \\
\hline$\ldots$ & $\ldots$ & $49183-49338$ & $43.32 \pm 0.08$ & $2115 \pm 575$ & $1251 \pm 72$ & 1 \\
\hline & & $49958-50112$ & $43.59 \pm 0.06$ & $1979 \pm 386$ & $1201 \pm 130$ & 1 \\
\hline Mrk 817 & 0.03145 & $49000-49212$ & $43.73 \pm 0.05$ & $3515 \pm 393$ & $1392 \pm 78$ & 1 \\
\hline$\ldots$ & $\ldots$ & $49404-49528$ & $43.61 \pm 0.05$ & $4952 \pm 537$ & $1971 \pm 96$ & 1 \\
\hline$\ldots$ & $\cdots$ & 49752-49924 & $43.61 \pm 0.05$ & $3752 \pm 995$ & $1729 \pm 158$ & 1 \\
\hline$\ldots$ & & $54200-54331$ & $43.78 \pm 0.05$ & $5627 \pm 30$ & $2025 \pm 5$ & 10 \\
\hline Mrk 1310 & 0.01956 & $54516-54618$ & $42.23 \pm 0.17$ & $1602 \pm 250$ & $755 \pm 138$ & 5,6 \\
\hline NGC 3227 & 0.00386 & $51480-54273$ & $42.24 \pm 0.11$ & $3578 \pm 83$ & $1376 \pm 44$ & 10 \\
\hline NGC 3516 & 0.00884 & $54181-54300$ & $42.73 \pm 0.21$ & $5175 \pm 96$ & $1591 \pm 10$ & 10 \\
\hline NGC 3783 & 0.00973 & $48607-48833$ & $42.55 \pm 0.18$ & $3093 \pm 529$ & $1753 \pm 141$ & 11 \\
\hline NGC 4051 & 0.00234 & $54180-54311$ & $41.96 \pm 0.20$ & $1034 \pm 41$ & $927 \pm 64$ & 12 \\
\hline NGC 4151 & 0.00332 & $53430-53471$ & $42.09 \pm 0.22$ & $4711 \pm 750$ & $2680 \pm 64$ & 13 \\
\hline NGC 4253 & 0.01293 & $54509-54618$ & $42.51 \pm 0.13$ & $834 \pm 1260$ & $516 \pm 91$ & 5,6 \\
\hline NGC 4593 & 0.00900 & $53391-53579$ & $42.87 \pm 0.18$ & $4141 \pm 416$ & $1561 \pm 55$ & 14 \\
\hline NGC 4748 & 0.01463 & $54505-54618$ & $42.49 \pm 0.13$ & $1212 \pm 173$ & $657 \pm 91$ & 5,6 \\
\hline NGC 5548 & 0.01717 & 47509-47809 & $43.33 \pm 0.10$ & $4044 \pm 199$ & $1687 \pm 56$ & 15 \\
\hline$\ldots$ & $\ldots$ & $47861-48179$ & $43.08 \pm 0.11$ & $4664 \pm 324$ & $1882 \pm 83$ & 15 \\
\hline$\ldots$ & $\cdots$ & $48225-48534$ & $43.29 \pm 0.10$ & $5776 \pm 237$ & $2075 \pm 81$ & 15 \\
\hline$\ldots$ & $\cdots$ & $48623-48898$ & $43.01 \pm 0.11$ & $5691 \pm 164$ & $2264 \pm 88$ & 15 \\
\hline$\ldots$ & $\cdots$ & 48954-49255 & $43.26 \pm 0.10$ & $2543 \pm 605$ & $1909 \pm 129$ & 15 \\
\hline$\ldots$ & $\ldots$ & $49309-49636$ & $43.32 \pm 0.10$ & $7202 \pm 392$ & $2895 \pm 114$ & 15 \\
\hline$\ldots$ & $\ldots$ & $49679-50008$ & $43.46 \pm 0.09$ & $6142 \pm 289$ & $2247 \pm 134$ & 15 \\
\hline$\cdots$ & $\cdots$ & $50044-50373$ & $43.37 \pm 0.09$ & $5706 \pm 357$ & $2026 \pm 68$ & 15 \\
\hline$\ldots$ & $\ldots$ & 50434-50729 & $43.18 \pm 0.10$ & $5541 \pm 354$ & $1923 \pm 62$ & 15 \\
\hline$\ldots$ & $\cdots$ & 50775-51085 & $43.52 \pm 0.09$ & $4596 \pm 505$ & $1732 \pm 76$ & 15 \\
\hline$\ldots$ & $\cdots$ & $51142-51456$ & $43.44 \pm 0.09$ & $6377 \pm 147$ & $1980 \pm 30$ & 15 \\
\hline$\ldots$ & $\ldots$ & $51517-51791$ & $43.05 \pm 0.11$ & $5957 \pm 224$ & $1969 \pm 48$ & 15 \\
\hline$\ldots$ & $\ldots$ & $51878-52174$ & $43.05 \pm 0.11$ & $6247 \pm 343$ & $2173 \pm 89$ & 15 \\
\hline$\ldots$ & $\cdots$ & $53431-53471$ & $42.90 \pm 0.13$ & $8047 \pm 1268$ & $3078 \pm 197$ & 16 \\
\hline$\ldots$ & $\cdots$ & $54508-54618$ & $42.95 \pm 0.11$ & $11177 \pm 2266$ & $4270 \pm 292$ & 5,6 \\
\hline$\ldots$ & & $54180-54332$ & $42.93 \pm 0.12$ & $4849 \pm 112$ & $1822 \pm 35$ & 10 \\
\hline NGC 6814 & 0.00521 & $54546-54618$ & $42.05 \pm 0.29$ & $3277 \pm 297$ & $1610 \pm 108$ & 5,6 \\
\hline PG $0026+129$ & 0.14200 & $48545-51084$ & $44.91 \pm 0.02$ & $1719 \pm 495$ & $1773 \pm 285$ & 17 \\
\hline PG $0052+251$ & 0.15500 & $48461-51084$ & $44.75 \pm 0.03$ & $4615 \pm 381$ & $1783 \pm 86$ & 17 \\
\hline PG $0804+761$ & 0.10000 & 48319-51085 & $44.85 \pm 0.02$ & $2012 \pm 845$ & $1971 \pm 105$ & 17 \\
\hline PG 0953+414 & 0.23410 & 48319-50997 & $45.13 \pm 0.01$ & $3002 \pm 398$ & $1306 \pm 144$ & 17 \\
\hline PG $1226+023$ & 0.15834 & 48361-50997 & $45.90 \pm 0.02$ & $2598 \pm 299$ & $1777 \pm 150$ & 17 \\
\hline PG 1229+204 & 0.06301 & 48319-50997 & $43.64 \pm 0.06$ & $3415 \pm 320$ & $1385 \pm 111$ & 17 \\
\hline PG $1307+085$ & 0.15500 & $48319-51042$ & $44.79 \pm 0.02$ & $5058 \pm 524$ & $1820 \pm 122$ & 17 \\
\hline PG $1411+442$ & 0.08960 & $48319-51038$ & $44.50 \pm 0.02$ & $2398 \pm 353$ & $1607 \pm 169$ & 17 \\
\hline PG $1426+015$ & 0.08647 & $48334-51042$ & $44.57 \pm 0.02$ & $6323 \pm 1295$ & $3442 \pm 308$ & 17 \\
\hline PG 1613+658 & 0.12900 & $48397-51073$ & $44.71 \pm 0.03$ & $7897 \pm 1792$ & $2547 \pm 342$ & 17 \\
\hline PG $1617+175$ & 0.11244 & $48362-51085$ & $44.33 \pm 0.02$ & $4718 \pm 991$ & $2626 \pm 211$ & 17 \\
\hline PG $1700+518$ & 0.29200 & $48378-51084$ & $45.53 \pm 0.03$ & $1846 \pm 682$ & $1700 \pm 123$ & 17 \\
\hline PG 2130+099 & 0.06298 & $55430-55557$ & $44.14 \pm 0.03$ & $2097 \pm 102$ & $1825 \pm 65$ & 2 \\
\hline SBS $1116+583 \mathrm{~A}$ & 0.02787 & $54505-54618$ & $42.07 \pm 0.28$ & $3604 \pm 1123$ & $1528 \pm 184$ & 5,6 \\
\hline
\end{tabular}

REFERENCES. - (1) Peterson et al. (1998); (2) Grier et al. (2012); (3) Dietrich et al. (1998); (4) Dietrich et al. (2012); (5) Bentz et al. (2009b); (6) Walsh et al. (2009); (7) Rodriguez-Pascual et al. (1997); (8) Santos-Lleó et al. (1997); (9) Santos-Lleó et al. (2001); (10) Denney et al. (2010); (11) Onken \& Peterson (2002); (12) Denney et al. (2009); (13) Bentz et al. (2006); (14) Denney et al. (2006); (15) Peterson et al. (2002) and references therein; (16) Bentz et al. (2007); (17) Kaspi et al. (2000). 
TABLE 2

Parameter Values for BLR Modeling.

\begin{tabular}{|c|c|c|c|c|c|c|c|}
\hline Object & $\begin{array}{c}R_{\mathrm{BLR}} \\
\text { (light day) }\end{array}$ & $\begin{array}{c}\theta_{\text {inc }} \\
\text { (degree) }\end{array}$ & $\begin{array}{c}\theta_{\text {opn }} \\
\text { (degree) }\end{array}$ & $\gamma$ & $\begin{array}{c}\log \tau_{\mathrm{d}} \\
\text { (day) }\end{array}$ & $f_{\mathrm{BLR}^{\mathrm{a}}}$ & Detrending ${ }^{b}$ \\
\hline $3 \mathrm{C} 120$ & $51.7 \pm 7.5$ & $43 \pm 22$ & $47 \pm 24$ & $-0.58 \pm 0.03$ & $2.34 \pm 0.31$ & $\ldots$ & $\mathrm{Y}$ \\
\hline$\ldots$ & $25.4 \pm 1.2$ & $49 \pm 21$ & $50 \pm 24$ & $-0.33 \pm 0.07$ & $1.66 \pm 0.44$ & $\ldots$ & $\ldots$ \\
\hline $3 C 390.3$ & $19.8 \pm 3.3$ & $42 \pm 25$ & $46 \pm 23$ & $-0.32 \pm 0.04$ & $3.08 \pm 0.42$ & $\ldots$ & $\ldots$ \\
\hline$\ldots$ & $41.0 \pm 4.1$ & $8 \pm 6$ & $12 \pm 9$ & $-0.06 \pm 0.16$ & $2.12 \pm 0.51$ & 15.4 & $\ldots$ \\
\hline Ark 120 & $36.6 \pm 6.3$ & $44 \pm 23$ & $47 \pm 24$ & $0.47 \pm 0.22$ & $1.83 \pm 0.69$ & $\ldots$ & $\cdots$ \\
\hline$\ldots$ & $30.8 \pm 10.2$ & $35 \pm 25$ & $40 \pm 23$ & $-0.65 \pm 0.11$ & $2.04 \pm 0.51$ & 1.3 & $\ldots$ \\
\hline Arp 151 & $4.0 \pm 0.4$ & $29 \pm 18$ & $36 \pm 22$ & $0.30 \pm 0.04$ & $1.88 \pm 0.59$ & 1.8 & $\cdots$ \\
\hline Fairall 9 & $38.6 \pm 5.7$ & $43 \pm 19$ & $49 \pm 24$ & $-0.66 \pm 0.02$ & $2.30 \pm 0.45$ & $\ldots$ & $\ldots$ \\
\hline Mrk 79 & $20.5 \pm 7.0$ & $42 \pm 23$ & $45 \pm 24$ & $-0.22 \pm 0.16$ & $2.29 \pm 0.55$ & $\cdots$ & $\ldots$ \\
\hline$\ldots$ & $21.2 \pm 3.9$ & $59 \pm 21$ & $43 \pm 24$ & $-0.05 \pm 0.12$ & $1.88 \pm 0.47$ & $\ldots$ & $\ldots$ \\
\hline$\ldots$ & $20.0 \pm 4.2$ & $24 \pm 21$ & $34 \pm 21$ & $-0.43 \pm 0.07$ & $2.28 \pm 0.49$ & 2.1 & $\ldots$ \\
\hline Mrk 110 & $22.7 \pm 6.4$ & $32 \pm 21$ & $42 \pm 22$ & $-0.57 \pm 0.10$ & $0.73 \pm 0.68$ & $\ldots$ & $\ldots$ \\
\hline$\ldots$ & $22.7 \pm 4.6$ & $36 \pm 24$ & $47 \pm 24$ & $-0.19 \pm 0.17$ & $1.18 \pm 0.38$ & $\ldots$ & $\mathrm{Y}$ \\
\hline$\ldots$ & $23.8 \pm 2.4$ & $14 \pm 9$ & $22 \pm 13$ & $-0.41 \pm 0.02$ & $2.68 \pm 0.44$ & 5.1 & $\ldots$ \\
\hline Mrk 142 & $15.3 \pm 2.7$ & $68 \pm 16$ & $45 \pm 24$ & $2.51 \pm 0.27$ & $0.82 \pm 0.36$ & $\cdots$ & $\ldots$ \\
\hline Mrk 202 & $4.9 \pm 1.4$ & $53 \pm 22$ & $51 \pm 23$ & $3.03 \pm 0.31$ & $1.04 \pm 0.42$ & $\ldots$ & $\ldots$ \\
\hline Mrk 279 & $18.4 \pm 4.7$ & $15 \pm 13$ & $20 \pm 16$ & $-0.38 \pm 0.05$ & $2.38 \pm 0.54$ & 5.3 & $\ldots$ \\
\hline Mrk 290 & $9.5 \pm 4.8$ & $24 \pm 12$ & $33 \pm 18$ & $-0.61 \pm 0.06$ & $2.15 \pm 0.54$ & 2.2 & $\ldots$ \\
\hline Mrk 335 & $15.9 \pm 2.5$ & $51 \pm 24$ & $47 \pm 24$ & $-0.25 \pm 0.11$ & $1.87 \pm 0.52$ & $\ldots$ & $\ldots$ \\
\hline$\ldots$ & $11.1 \pm 5.0$ & $42 \pm 23$ & $45 \pm 24$ & $-0.59 \pm 0.10$ & $1.45 \pm 0.48$ & $\ldots$ & $\ldots$ \\
\hline$\ldots$ & $15.8 \pm 0.8$ & $15 \pm 8$ & $24 \pm 11$ & $-0.29 \pm 0.02$ & $2.28 \pm 0.45$ & 4.4 & $\ldots$ \\
\hline Mrk 509 & $108.9 \pm 4.0$ & $68 \pm 11$ & $34 \pm 21$ & $-0.19 \pm 0.03$ & $2.46 \pm 0.27$ & 0.8 & $\ldots$ \\
\hline Mrk 590 & $23.5 \pm 6.2$ & $30 \pm 19$ & $39 \pm 26$ & $0.21 \pm 0.22$ & $1.96 \pm 0.61$ & 1.5 & $\ldots$ \\
\hline$\ldots$ & $16.5 \pm 3.6$ & $48 \pm 24$ & $48 \pm 24$ & $2.25 \pm 0.23$ & $2.21 \pm 0.54$ & $\ldots$ & $\ldots$ \\
\hline$\ldots$ & $30.3 \pm 4.3$ & $30 \pm 21$ & $33 \pm 24$ & $1.10 \pm 0.35$ & $1.91 \pm 0.55$ & 1.9 & $\ldots$ \\
\hline$\ldots$ & $28.8 \pm 3.6$ & $23 \pm 18$ & $32 \pm 23$ & $-0.06 \pm 0.14$ & $2.24 \pm 0.53$ & 2.3 & $\ldots$ \\
\hline Mrk 817 & $19.4 \pm 2.6$ & $15 \pm 13$ & $26 \pm 19$ & $-0.03 \pm 0.20$ & $1.27 \pm 0.42$ & 4.0 & $\mathrm{Y}$ \\
\hline$\ldots$ & $19.0 \pm 2.7$ & $41 \pm 22$ & $45 \pm 23$ & $0.14 \pm 0.16$ & $1.92 \pm 0.59$ & $\ldots$ & $\ldots$ \\
\hline$\ldots$ & $36.1 \pm 9.1$ & $37 \pm 24$ & $39 \pm 26$ & $0.16 \pm 0.22$ & $1.85 \pm 0.48$ & 1.3 & $\ldots$ \\
\hline$\ldots$ & $10.7 \pm 1.2$ & $57 \pm 21$ & $47 \pm 24$ & $0.25 \pm 0.15$ & $1.67 \pm 0.29$ & $\cdots$ & $\mathrm{Y}$ \\
\hline Mrk 1310 & $4.3 \pm 0.5$ & $11 \pm 9$ & $18 \pm 14$ & $0.69 \pm 0.12$ & $0.95 \pm 0.18$ & 7.6 & $\mathrm{Y}$ \\
\hline NGC 3227 & $5.1 \pm 0.7$ & $60 \pm 22$ & $48 \pm 24$ & $0.27 \pm 0.11$ & $0.42 \pm 0.13$ & $\ldots$ & $\mathrm{Y}$ \\
\hline NGC 3516 & $15.3 \pm 1.2$ & $61 \pm 13$ & $36 \pm 24$ & $-0.25 \pm 0.05$ & $2.17 \pm 0.57$ & 0.9 & $\ldots$ \\
\hline NGC 3783 & $5.8 \pm 3.6$ & $43 \pm 24$ & $47 \pm 24$ & $-0.07 \pm 0.13$ & $1.81 \pm 0.58$ & $\ldots$ & $\ldots$ \\
\hline NGC 4051 & $2.5 \pm 0.7$ & $73 \pm 14$ & $36 \pm 24$ & $-0.01 \pm 0.06$ & $0.82 \pm 0.20$ & 0.8 & $\ldots$ \\
\hline NGC 4151 & $7.9 \pm 0.9$ & $53 \pm 18$ & $57 \pm 21$ & $0.22 \pm 0.05$ & $1.89 \pm 0.62$ & $\ldots$ & $\ldots$ \\
\hline NGC 4253 & $7.1 \pm 1.6$ & $43 \pm 23$ & $47 \pm 23$ & $0.41 \pm 0.16$ & $0.09 \pm 0.20$ & $\ldots$ & $\ldots$ \\
\hline NGC 4593 & $4.3 \pm 0.7$ & $36 \pm 24$ & $42 \pm 23$ & $-0.18 \pm 0.12$ & $1.13 \pm 0.39$ & $\ldots$ & $\ldots$ \\
\hline NGC 4748 & $7.3 \pm 1.0$ & $58 \pm 21$ & $50 \pm 23$ & $0.43 \pm 0.11$ & $0.79 \pm 0.27$ & $\ldots$ & $\ldots$ \\
\hline NGC 5548 & $21.9 \pm 0.8$ & $9 \pm 6$ & $15 \pm 8$ & $-0.24 \pm 0.03$ & $1.92 \pm 0.40$ & 11.2 & $\ldots$ \\
\hline ... & $18.8 \pm 1.1$ & $19 \pm 12$ & $29 \pm 18$ & $0.48 \pm 0.05$ & $2.57 \pm 0.51$ & 3.0 & $\ldots$ \\
\hline$\ldots$ & $16.7 \pm 1.6$ & $29 \pm 19$ & $38 \pm 23$ & $0.23 \pm 0.12$ & $1.52 \pm 0.41$ & 1.6 & $\ldots$ \\
\hline$\ldots$ & $12.9 \pm 1.2$ & $62 \pm 19$ & $44 \pm 25$ & $1.07 \pm 0.07$ & $2.46 \pm 0.45$ & $\ldots$ & $\mathrm{Y}$ \\
\hline$\ldots$ & $14.3 \pm 0.8$ & $13 \pm 8$ & $19 \pm 11$ & $-0.31 \pm 0.05$ & $1.66 \pm 0.31$ & 6.3 & $\mathrm{Y}$ \\
\hline$\ldots$ & $15.2 \pm 1.4$ & $63 \pm 16$ & $46 \pm 24$ & $0.21 \pm 0.04$ & $2.05 \pm 0.43$ & $\ldots$ & $\ldots$ \\
\hline$\ldots$ & $19.9 \pm 1.4$ & $55 \pm 20$ & $47 \pm 25$ & $-0.09 \pm 0.05$ & $2.17 \pm 0.49$ & $\ldots$ & $\ldots$ \\
\hline$\ldots$ & $16.6 \pm 0.8$ & $17 \pm 9$ & $23 \pm 12$ & $-0.25 \pm 0.03$ & $2.47 \pm 0.43$ & 4.2 & $\ldots$ \\
\hline$\ldots$ & $20.4 \pm 1.6$ & $47 \pm 21$ & $53 \pm 24$ & $0.19 \pm 0.07$ & $1.38 \pm 0.38$ & $\ldots$ & $\ldots$ \\
\hline$\ldots$ & $29.4 \pm 1.5$ & $42 \pm 18$ & $59 \pm 20$ & $-0.20 \pm 0.04$ & $2.00 \pm 0.40$ & $\ldots$ & $\ldots$ \\
\hline$\ldots$ & $24.4 \pm 2.9$ & $48 \pm 21$ & $50 \pm 23$ & $-0.39 \pm 0.05$ & $1.54 \pm 0.38$ & $\ldots$ & $\ldots$ \\
\hline$\ldots$ & $8.2 \pm 2.1$ & $61 \pm 21$ & $43 \pm 24$ & $0.15 \pm 0.11$ & $0.87 \pm 0.31$ & $\ldots$ & $\ldots$ \\
\hline$\ldots$ & $19.5 \pm 2.4$ & $72 \pm 15$ & $33 \pm 22$ & $0.89 \pm 0.15$ & $1.20 \pm 0.30$ & 0.8 & $\ldots$ \\
\hline$\ldots$ & $5.9 \pm 1.3$ & $40 \pm 23$ & $46 \pm 23$ & $0.94 \pm 0.25$ & $1.05 \pm 0.74$ & $\ldots$ & $\ldots$ \\
\hline$\ldots$ & $5.3 \pm 0.9$ & $65 \pm 20$ & $43 \pm 25$ & $0.08 \pm 0.07$ & $1.56 \pm 0.60$ & $\ldots$ & $\ldots$ \\
\hline$\ldots$ & $3.9 \pm 0.9$ & $54 \pm 23$ & $48 \pm 24$ & $1.46 \pm 0.11$ & $2.13 \pm 0.56$ & $\ldots$ & $\mathrm{Y}$ \\
\hline NGC 6814 & $6.9 \pm 0.7$ & $56 \pm 21$ & $55 \pm 23$ & $-0.03 \pm 0.08$ & $0.95 \pm 0.34$ & $\ldots$ & $\mathrm{Y}$ \\
\hline PG $0026+129$ & $136.4 \pm 32.2$ & $56 \pm 21$ & $48 \pm 24$ & $-0.41 \pm 0.05$ & $2.37 \pm 0.27$ & $\ldots$ & $\ldots$ \\
\hline PG $0052+251$ & $104.1 \pm 8.0$ & $56 \pm 20$ & $44 \pm 26$ & $-0.24 \pm 0.06$ & $2.45 \pm 0.30$ & $\ldots$ & $\ldots$ \\
\hline PG $0804+761$ & $109.4 \pm 7.5$ & $27 \pm 12$ & $32 \pm 20$ & $-0.53 \pm 0.03$ & $2.54 \pm 0.28$ & 2.1 & $\mathrm{Y}$ \\
\hline PG $0953+414$ & $143.1 \pm 28.6$ & $38 \pm 20$ & $37 \pm 23$ & $-0.28 \pm 0.09$ & $2.24 \pm 0.29$ & 1.4 & $\mathrm{Y}$ \\
\hline PG $1226+023$ & $392.5 \pm 45.7$ & $58 \pm 20$ & $45 \pm 25$ & $0.10 \pm 0.12$ & $2.67 \pm 0.33$ & $\ldots$ & $\ldots$ \\
\hline PG $1229+204$ & $36.0 \pm 6.6$ & $53 \pm 23$ & $47 \pm 24$ & $0.36 \pm 0.13$ & $2.01 \pm 0.23$ & $\ldots$ & $\ldots$ \\
\hline PG $1307+085$ & $120.6 \pm 30.1$ & $54 \pm 23$ & $46 \pm 24$ & $0.34 \pm 0.18$ & $1.94 \pm 0.22$ & $\ldots$ & $\ldots$ \\
\hline PG $1411+442$ & $45.6 \pm 12.5$ & $36 \pm 25$ & $48 \pm 24$ & $-0.44 \pm 0.09$ & $1.62 \pm 0.22$ & $\ldots$ & $\ldots$ \\
\hline PG $1426+015$ & $122.4 \pm 25.5$ & $46 \pm 22$ & $48 \pm 24$ & $-0.30 \pm 0.09$ & $2.84 \pm 0.34$ & $\cdots$ & $\cdots$ \\
\hline PG $1613+658$ & $137.1 \pm 8.1$ & $24 \pm 10$ & $19 \pm 15$ & $-0.27 \pm 0.08$ & $2.83 \pm 0.33$ & 3.7 & $\ldots$ \\
\hline PG $1617+175$ & $116.4 \pm 23.9$ & $47 \pm 21$ & $51 \pm 24$ & $-0.30 \pm 0.07$ & $2.48 \pm 0.27$ & $\ldots$ & $\ldots$ \\
\hline PG $1700+518$ & $372.4 \pm 73.2$ & $55 \pm 23$ & $51 \pm 24$ & $-0.14 \pm 0.17$ & $2.16 \pm 0.29$ & $\ldots$ & $\mathrm{Y}$ \\
\hline PG 2130+099 & $32.7 \pm 3.0$ & $51 \pm 5$ & $9 \pm 8$ & $-0.20 \pm 0.06$ & $2.43 \pm 0.59$ & 1.6 & $\ldots$ \\
\hline SBS $1116+583 A$ & $3.3 \pm 0.8$ & $52 \pm 23$ & $49 \pm 23$ & $0.56 \pm 0.15$ & $0.45 \pm 0.22$ & $\ldots$ & $\cdots$ \\
\hline
\end{tabular}

${ }^{\text {a }}$ Only $f_{\mathrm{BLR}}$ for $\theta_{\mathrm{opn}}<40^{\circ}$ is calculated using Equation (22).

b " $\mathrm{Y}$ " means detrending is implemented. 